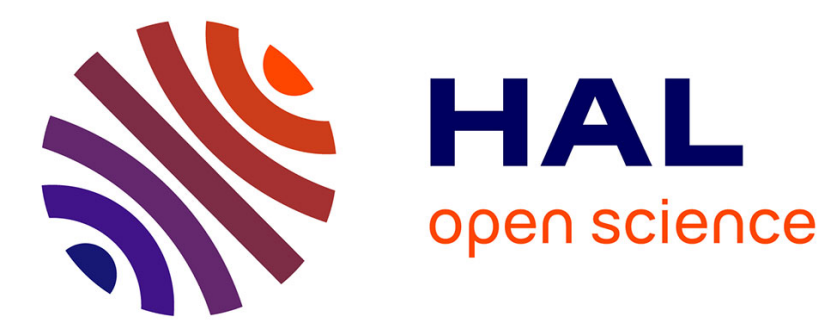

\title{
A new route to $\alpha$-fluorovinylphosphonates utilizing Peterson olefination methodology
} Rachel Waschbüsch, John Carran, Philippe Savignac

\section{To cite this version:}

Rachel Waschbüsch, John Carran, Philippe Savignac. A new route to $\alpha$-fluorovinylphosphonates utilizing Peterson olefination methodology. Tetrahedron, 1996, 52 (45), pp.14199-14216. 10.1016/00404020(96)00859-9 . hal-03166546

\section{HAL Id: hal-03166546 https://hal.science/hal-03166546}

Submitted on 11 Mar 2021

HAL is a multi-disciplinary open access archive for the deposit and dissemination of scientific research documents, whether they are published or not. The documents may come from teaching and research institutions in France or abroad, or from public or private research centers.
L'archive ouverte pluridisciplinaire HAL, est destinée au dépôt et à la diffusion de documents scientifiques de niveau recherche, publiés ou non, émanant des établissements d'enseignement et de recherche français ou étrangers, des laboratoires publics ou privés. 


\title{
A New Route to $\alpha$-Fluorovinylphosphonates Utilizing Peterson Olefination Methodology
}

\author{
Rachel Waschbüsch, John Carran and Philippe Savignac \\ Hétéroéléments et Coordination, URA CNRS 1499, DCPH, Ecole Polytechnique, 91128 Palaiseau Cedex., France. \\ Fax. (+33) 0169333990
}

\begin{abstract}
Several $\alpha$-fluorovinylphosphonates (6) have been synthesised from the Peterson olefination reaction applied to both aldehydes and ketones in conjunction with $\alpha$-lithiated- $\alpha$-fluoro- $\alpha$ trimethylsilylmethylphosphonate (2). The reaction with aldehydes gives mainly the $E$-isomer whereas reaction with ketones gives mainly the $Z$-isomer. We propose a closed transition state to explain the results of our study.
\end{abstract}

\section{INTRODUCTION}

Phosphonic acids exhibit important biological properties because of their similarity to phosphates. ${ }^{1}$ The carbon-phosphorus bond in phosphonates, unlike phosphates, is not susceptible to the hydrolytic action of phosphatases, thereby imparting greater stability under physiological conditions. In pioneering work Blackburn et al. established that $\alpha$-fluorination of phosphonates is a successful strategy for the design of phosphonate analogues of phosphate esters. ${ }^{2}$ Due to this concept the use of $\alpha$-fluorophosphonates has become a field of considerable importance in modern organophosphorus chemistry. To develop the utility of this concept Blackburn and Parratt ${ }^{2 c}$ describe, among others, a new route to $\alpha$-fluoroalkylphosphonates via the catalytic reduction of $\alpha$-fluorovinylphosphonates. They reported the synthesis of $\alpha$-fluorovinylphosphonates based on a Wittig-Horner condensation of the tetraisopropyl ester of fluoromethylenebisphosphonic acid with aldehydes and ketones. With aldehydes the less hindered $E$-isomer was mainly obtained whereas with ketones a lower stereoselectivity was observed and the major isomer appears to have the phosphoryl group trans to the more bulky ketone substituent.

However, this Wittig-Horner olefination methodology did not allow access to the $Z$-isomer. The problem was recently solved by utilizing a stereoselective palladium (0) coupling reaction between a gem fluoroiodoalkene and diethyl phosphite; this method complements the Wittig-Horner olefination route. ${ }^{3}$ Even though the stereoselective construction of $\alpha$-fluorovinylphosphonates may have broad general applicability, there are relatively few methods available for the synthesis of the $Z$-isomer.

Recently, we described a new and successful methodology 4 for the preparation of $\alpha$ fluoroalkylphosphonates employing the $\alpha$-lithiated- $\alpha$-fluoro- $\alpha$-trimethylsilylmethylphosphonate (2) which was prepared from diethyl dibromofluoromethylphosphonate (1) and chlorotrimethylsilane. Armed with this knowledge we thought that this methodology could be used to promote a new version of the Peterson 
olefination reaction and consequently help us to develop a new route to $\alpha$-fluorovinylphosphonates (6). The Peterson olefination reaction is a powerful alternative to Wittig-Horner olefination ${ }^{5}$ as less reactive carbonyl compounds (such as ketones) are tolerated leading to a wider range of available alkenes. ${ }^{6}$

Due to the mild conditions required for the formation of $\mathbf{2}$, the Peterson olefination route, described herein, should be a potentially useful alternative to the Wittig-Horner reaction in the synthesis of $\alpha$ fluorovinylphosphonates. The trimethylsilyl group, previously introduced as a passive protecting group to warrant clean alkylation reactions of the lithium derivative $\mathbf{2}$, could participate in the reaction actively affecting stereoselectivity.

\section{RESULTS AND DISCUSSION}

Diethyl dibromofluoromethylphosphonate 1 was prepared by reaction of triethylphosphite, (EtO) ${ }_{3} \mathrm{P}$, with fluorotribromomethane according to Michaelis-Arbuzov conditions. It has been demonstrated that among all the mechanistic pathways (ionic, radical, monoelectronic transfer) which can be involved for this type of reaction, the halophilic substitution $\left(\mathrm{S}_{\mathrm{N}} \mathrm{X}^{+}\right.$, with $\left.\mathrm{X}=\mathrm{Cl}, \mathrm{Br}\right)$ is the major contribution. ${ }^{7}$ Burton and Flynn ${ }^{8}$ obtained 1 in good yield $\left(78 \%\right.$ ) by reaction between $(\mathrm{EtO})_{3} \mathrm{P}$ and $\mathrm{CFBr}_{3}$ in refluxing diethyl ether. For our part after several experiments in various solvents ( $\mathrm{Et}_{2} \mathrm{O}$, THF, hexane) and at different temperatures we chose to operate in hexane at room temperature. The reaction time was lengthy (11 days) but due to the mild conditions the quantity of side-products was considerably reduced ((EtO) ${ }_{3} \mathrm{PO}$ was the main side-product) and the crude product could be used without purification (Scheme 1).

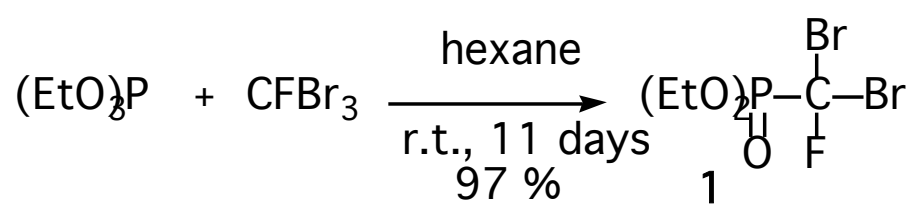

Scheme 1.

The $\alpha$-lithiated- $\alpha$-fluoro- $\alpha$-trimethylsilylmethylphosphonate (2) was readily and quantitatively prepared from diethyl dibromofluoromethylphosphonate (1) and chlorotrimethylsilane as the trapping agent, under internal quench conditions. Our logic was to minimise the time between lithiation and reaction with the electrophile and accordingly to inhibit the decomposition of the intermediate $\alpha$-lithiated $\alpha$ halogenophosphonate. For that reason chlorotrimethylsilane (1 equiv) and 1 (1 equiv) in THF solution were simultaneously added at low temperature $\left(-78^{\circ} \mathrm{C}\right)$ to $n$-butyllithium $(2.1$ equiv) diluted in an equal volume of THF. The combination of double lithium-bromine exchange and silylation reactions was exothermic and the temperature had to be carefully controlled in order to prevent alkylation of the $\alpha$-lithiated- $\alpha$-fluoro- $\alpha$ trimethylsilylmethylphosphonate (2) by simultaneously formed $n$-butylbromide ( 2 equiv). For that reason all the reactions of carbanion $\mathbf{2}$ here described were conducted at low temperature.

In order to simplify the access to $\alpha$-fluorovinylphosphonate (6) several attempts were made to obtain the necessary $\alpha$-fluoro- $\alpha$-trimethylsilylmethylphosphonate (3) on a large scale. Unfortunately under all conditions examined the protonation of the carbanion 2 with formic acid at low temperature followed by careful treatment in aqueous acidic medium was ineffective at promoting the formation of $\mathbf{3}$ in pure form 
(Scheme 2). The silylated compound $\mathbf{3}$ was isolated as the major product but it was generally contaminated with the desilylated compound $(4,8-10 \%)$ presumably as a result of the sensitivity of the C-Si bond to hydrolysis afforded by the fluorine atom. In light of these last results we undertook a study on the use of $\mathbf{1}$ as precursor to $\alpha$-fluorovinylphosphonates (6).<smiles>CCO[Pb](=O)C(F)(Br)Br</smiles>

1

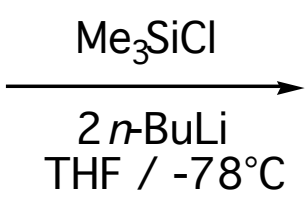

$\mathrm{THF} /-78^{\circ} \mathrm{C}$<smiles>CCO[Pb](=O)C(F)([SiH3])[SiH2][TeH]</smiles>

2<smiles>CCO[PH3+]C(=O)OCC</smiles>

3

4

\section{Scheme 2.}

At low temperature $\left(-78^{\circ} \mathrm{C}\right)$ in THF, 2 cleanly underwent aldol condensation with aldehydes and ketones. The reaction has been performed on a variety of structurally different carbonyl compounds to determine the scope and limitations of this method. The formation of $\alpha$-fluorovinylphosphonates (6) was immediate and, on account of the presence of a carbanion-stabilizing group $\left((\mathrm{EtO})_{2} \mathrm{PO}\right)$ on the carbon-bearing silicon, we were unable to isolate the $\beta$-hydroxysilanes and the $\alpha$-fluorovinylphosphonates (6, usually an $E$ - $Z$ mixture) were isolated directly from the reaction (Scheme 3). Under these conditions the Peterson olefination reaction exclusively occurred and no trace of the Wittig-Horner reaction was detected.<smiles>CCOP(=O)(OC)C(F)(Br)Br</smiles><smiles>[Z10]OP(=O)(OC)C(F)=C([R])[R]</smiles>

$6 E / 6-Z$

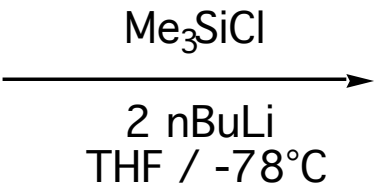

- MezSiOLi

Scheme 3.

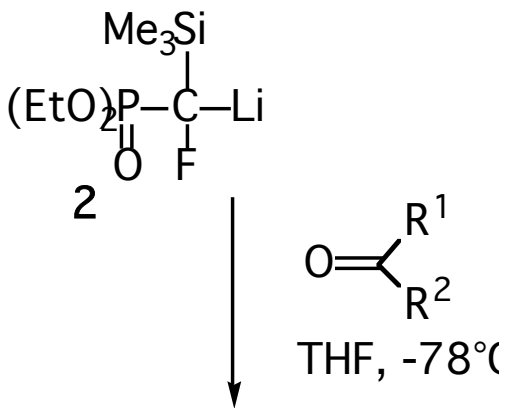

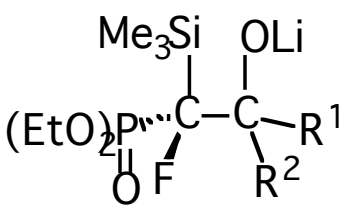

5 A 5 B

The so formed $\beta$-oxidosilane intermediates (5) have been shown to undergo $\beta$-elimination to form olefins by a syn elimination mechanism under basic conditions. ${ }^{9}$ By analogy to the Wittig-Horner reaction, 
the reaction pathway is assumed to involve a 4-centered species. Of the two configurations (A and $\mathbf{B})$ of $\beta$-oxidosilanes (5) which have the syn alignment of alkoxide and silicon, $\mathbf{A}$ with $\mathrm{R}^{1}=\mathrm{H}$ or $\mathrm{R}^{1}<\mathrm{R}^{2}$ (which would lead to the trans isomer) is preferable on steric grounds ${ }^{10}$ (Scheme 4).
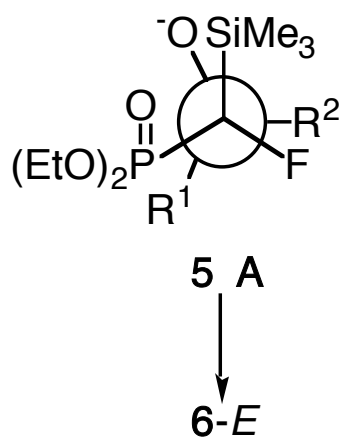
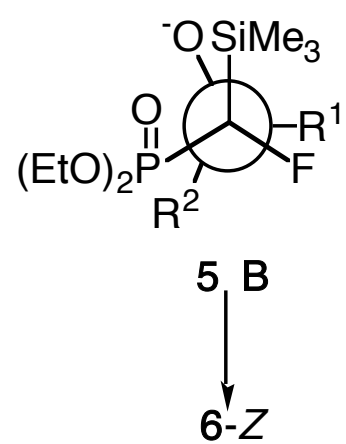

\section{Scheme 4.}

Some results are summarized in Table 1 and as shown the reaction was exceptionally effective for nonenolizable carbonyl compounds, giving a variety of $\alpha$-fluorovinylphosphonates $(6)$ in excellent yields. They were stable under the reaction conditions and the $E / Z$ isomer ratios were unchanged before and after isolation from the reaction, indicating that isomerization does not occur during workup. The selectivity observed by ${ }^{31} \mathrm{P}$ NMR spectroscopy was equivalent to that reported in Table 1.

The selectivity of the Peterson olefination reaction is directly related to the diastereoselectivity of the aldol process which may in turn be dependent upon the phosphonate carbanion environment, which is extremely difficult to control. There are a number of methods for the synthesis of diastereomerically pure $\beta$-hydroxysilanes, ${ }^{11}$ however, the majority of these procedures do not involve a carbon-carbon bond forming step between the two atoms that will comprise the double bond. A general and consistent method of preparing diastereoisomerically pure $\beta$-hydroxysilanes is via the use of $\alpha$-silyl ketone ${ }^{11}$ although the latter are rarely used since there are few general and high yielding methods for their production.

As shown in Table 1, reaction between 2 and aliphatic aldehydes generally furnished a mixture of $E$ and $Z$-isomers in nearly equal amounts (6a-d) corresponding to an equivalent probability of formation of the two configurations $\mathbf{5 A}$ and $\mathbf{5 B}$. With aromatic or heteroaromatic aldehydes the $E$ isomers were produced as main products $(\mathbf{6 e - k})$ corresponding to a preference for configuration $\mathbf{5 A}$. When the reaction of $\mathbf{2}$ was extended to ketones the composition products seemed to be dependent on steric factors and the respective $Z$ isomers were produced as main products (6--t) corresponding to a preference for configuration 5B. Particularly noteworthy is also the fact that the reaction between $\mathbf{2}$ and cyclic ketones only produced Zisomers (6p-s). By contrast, cyclic ketones bearing bulky groups in the $\alpha$-position (2,2dimethylcyclohexanone, 2-t-butylcyclohexanone, (R)-(+)-pulegone and isopropenylmethyl ketone) were completely enolized. To rationalize all these results it is possible that when the $\beta$-oxidosilanes $\mathbf{5}$ are generated the elimination step takes place faster than a conformational change. Each isomer of product $\mathbf{6}$ reported in Table 1 was isolated and purified by column chromatography and fully characterized by NMR $\left({ }^{31} \mathrm{P},{ }^{1} \mathrm{H}\right.$ and ${ }^{13} \mathrm{C}$ ) and mass spectrometry. 


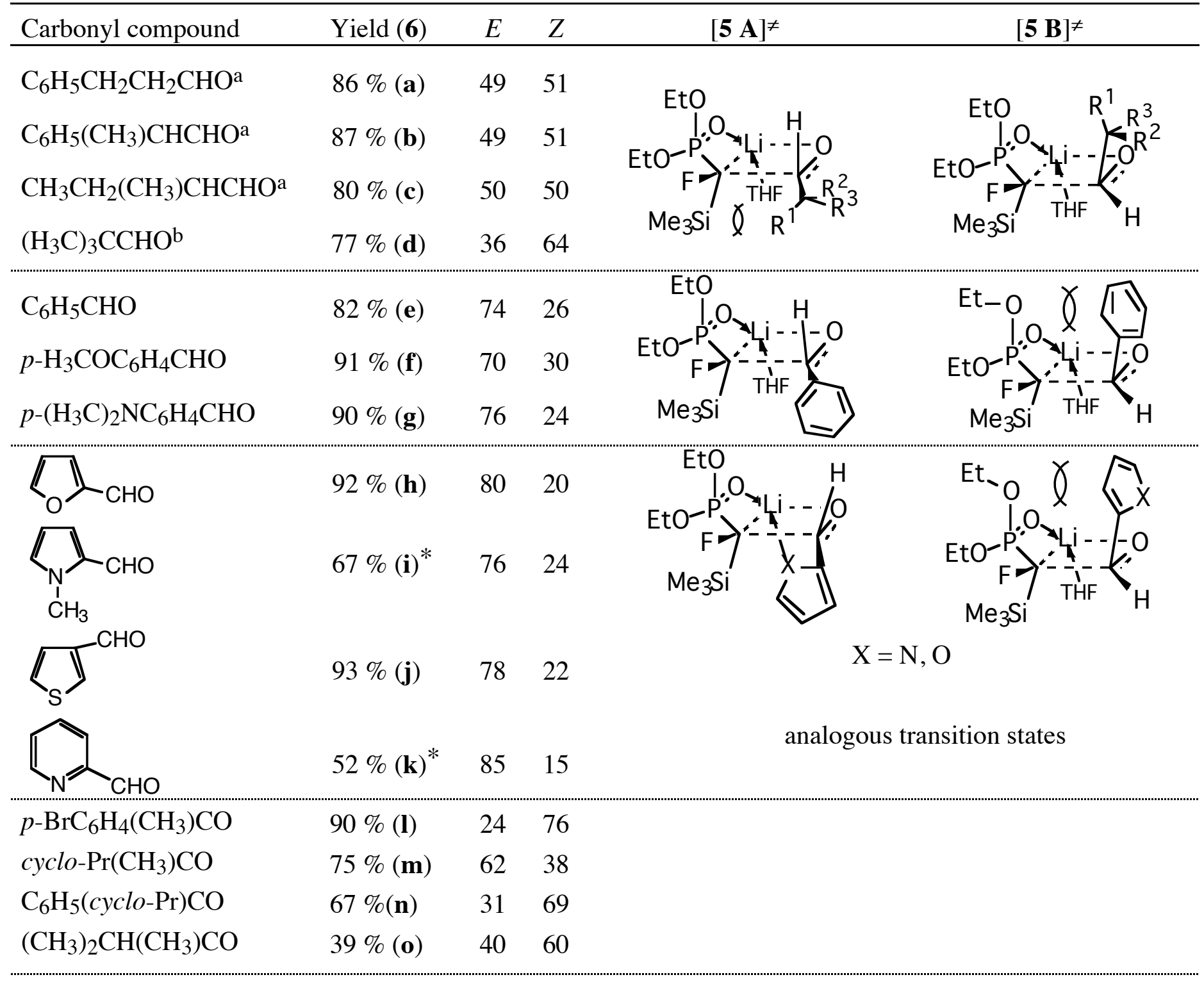

$\begin{array}{llll}(-) \text {-Carvone }^{c} & 87 \%(\mathbf{p}) & 0 & 100 \\ \text { Isophorone }^{\mathrm{d}} & 84 \%(\mathbf{q}) & 0 & 100 \\ \alpha \text {-Tetralone }^{\mathrm{e}} & 52 \%(\mathbf{r}) & 0 & 100\end{array}$
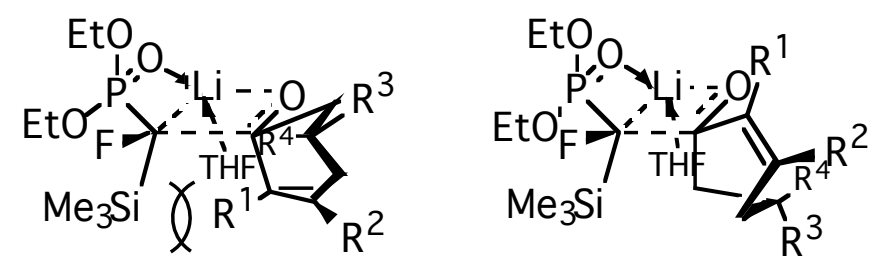<smiles>CC1CC[I-]CC1=O</smiles>

$62 \%(\mathbf{s}) \quad 0 \quad 100$
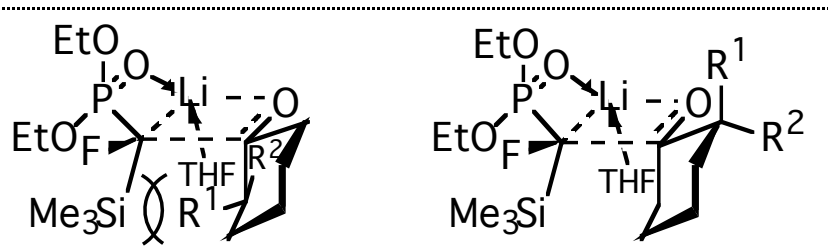<smiles>COC1CC[C+]CC1=O</smiles>

$80 \%(\mathbf{t}) \quad 24 \quad 76$
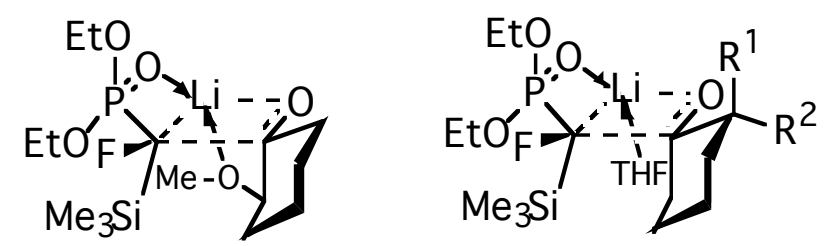

\footnotetext{
* Quantitative crude yield

a $\mathrm{R}^{1}=\mathrm{H}, \mathrm{R}^{2} / \mathrm{R}^{3}=\mathrm{H}$ or alkyl, no interaction

$\mathrm{b}^{1}=\mathrm{R}^{2}=\mathrm{R}^{3}=\mathrm{CH}_{3}$

c $\mathrm{R}^{1}=\mathrm{CH}_{3}, \mathrm{R}^{2}=\mathrm{R}^{4}=\mathrm{H}, \mathrm{R}^{3}=i-\mathrm{Pr}$
}

${ }^{\mathrm{d}} \mathrm{R}^{1}=\mathrm{H}, \mathrm{R}^{2}=\mathrm{R}^{3}=\mathrm{R}^{4}=\mathrm{CH}_{3}$

e $\mathrm{R}^{1}, \mathrm{R}^{2}=\mathrm{HC}=\mathrm{CH}-\mathrm{CH}=\mathrm{CH}, \mathrm{R}^{3}=\mathrm{R}^{4}=\mathrm{H}$

${ }^{f} \mathrm{R}^{1}=\mathrm{H}$ and $\mathrm{R}^{2}=\mathrm{CH}_{3}$ or $\mathrm{R}^{1}=\mathrm{CH}_{3}$ and $\mathrm{R}^{2}=\mathrm{H}$

$\mathrm{g} \mathrm{R}^{1}=\mathrm{H}$ and $\mathrm{R}^{2}=\mathrm{OCH}_{3}$ or $\mathrm{R}^{1}=\mathrm{OCH}_{3}$ and $\mathrm{R}^{2}=\mathrm{H}$ 
Thus to illustrate our results (Table 1), we tentatively propose a mechanistic pathway which implies a closed transition state of the type proposed by Warren et al. for $\alpha$-lithiated diaryl methylphosphine oxide in reaction with formaldehyde. ${ }^{12}$

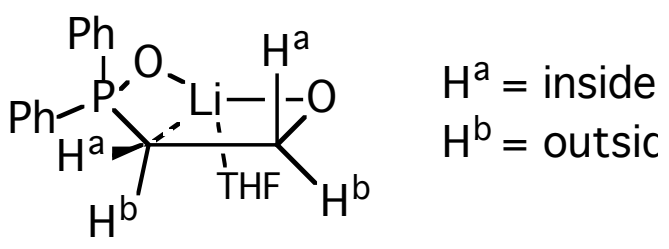

In this diagram Warren et al. annotate $\mathrm{H}^{\mathrm{a}}$ as inside hydrogens and $\mathrm{H}^{\mathrm{b}}$ as outside ones and these positions are attributed from their spatial orientation in the "butterfly" like transition state.

In the case of our $\alpha$-lithiated- $\alpha$-fluoro- $\alpha$-trimethylsilylmethylphosphonates (2) in reaction with carbonyl compounds, the bulkier trimethylsilyl group replaces the outside hydrogen $\left(\mathrm{H}^{\mathrm{b}}\right)$.

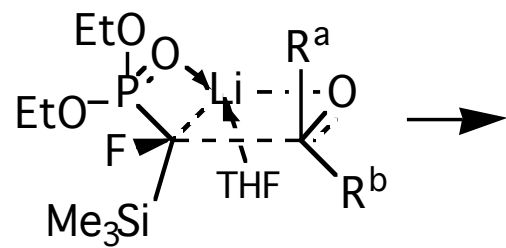

$\left[5 \mathrm{~A}^{ \pm} / \$ B\right]^{ \pm}$

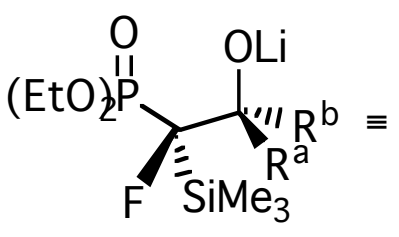

$5 \mathrm{~A} / 5 \mathrm{~B}$

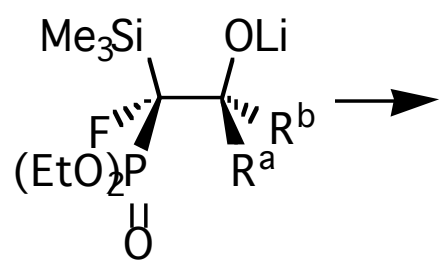

O

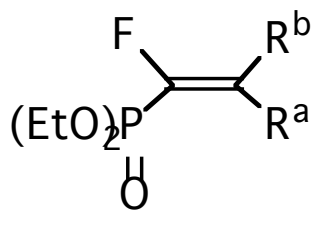

$6(E) / 6(Z)$

Scheme 5.

This representation (Scheme 5) allows us to explain most of our results. With aliphatic aldehydes, the transition states $[\mathbf{5 A}]^{\neq}$and $[\mathbf{5 B}]^{\neq}$, respectively yielding $\beta$-oxidosilanes $\mathbf{5 A}$ and $\mathbf{5 B}$, show no steric interactions (6a-c, $E / Z$ ratio 50/50) in cases where the carbon linked to the carbonyl group bears one or more protons. Homoaromatic aldehydes preferentially take the transition state $[\mathbf{5 A}]^{\neq}$since the phenyl groups orientate in the outside position which preferentially generates the $E$-isomer (6e-g, $E / Z$ ratio 70/30 - 76/24). Heteroaromatic aldehydes with an heteroatom $(\mathrm{O}, \mathrm{N}, \mathrm{S})$ in the $\alpha$ or $\beta$-position increase the $E$ to $Z$ olefin ratio $(\mathbf{6 h}-\mathbf{k}, E / Z$ ratio $76 / 24$ - 85/20). This is probably due to an intramolecular interaction between the heteroatom and the lithium which is much more favorable than the intermolecular interaction between the THF and the lithium. In the case of $\alpha, \beta$-unsaturated cyclohexanone derivatives and $\alpha$-substituted cyclohexanone, the transition state [5. $]^{\neq}$ shows a major sterical hindrance between $\mathrm{R}^{1}$ and the silyl group which explains the obtention of only the $Z$ isomer in the case of $\mathbf{6 p - s}$. The presence of an heteroatom in the $\alpha$-position of the carboxyl group favours an interaction with the lithium despite the previously stated sterical hindrance, this explains the small amount of $E$ isomer obtained in the case of $\mathbf{6 t}$ ( $E / Z$ ratio 24/76). However, this representation of the intermediate [5] $]^{\neq}$ does not allow us to explain the $E / Z$ ratio obtained with the acyclic ketones since it is not easy to compare the size of the $\alpha$-substituents of the ketones 6l-o.

The elimination step in the Peterson olefination reaction is affected by various factors such as aggregation, ion pairing or solvation. In order to determine how the elimination step was affected by steric factors, we have additionally investigated this reaction with some lithiated phosphonates of type $\mathbf{2}$ bearing a variety of structurally different alkylsilanes on the $\alpha$ carbon according to the methodology described in Scheme 3. We attempted two types of experiments: 
Firstly with a carbonyl compound that gave $\mathrm{E}$ as the major isomer. As shown in Table 2, the reactions in THF between $\alpha$-lithiated- $\alpha$-fluoro- $\alpha$-trialkylsilylmethylphosphonates (2) and $p$-anisaldehyde, taken as a model, work well to afford $\mathbf{6 f}$ in excellent yield (90\%). In agreement with the axial orientation of the trialkylsilyl group in the "butterfly" like transition state, the $E / Z$ ratio decreases on increasing steric bulk of silyl reagent until a critical size whereupon the reaction course is modified. When the reaction was performed with chlorotriisopropylsilane the major product $(50 \%)$ was the expected $\alpha$-fluorovinylphosphonates $6 \mathbf{6}$, in an $E / Z$ ratio $(98 / 2)$, but it was obtained together with the $\alpha$-fluoro- $\alpha$-silyl alkenes $(7,33 \%)$ formed from a WittigHorner olefination side reaction (Scheme 6).
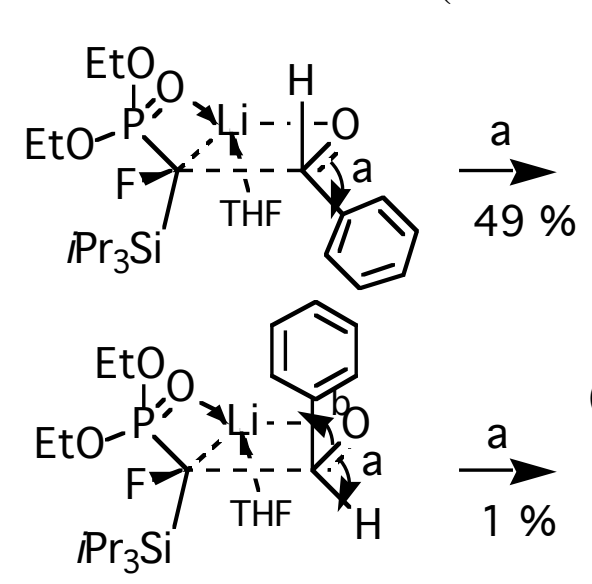<smiles>CCOP(=O)(OCC)C(OCl)(c1ccccc1)C(C)(C)C(F)(F)F</smiles><smiles>CCOC(=O)/C(F)=C/c1ccccc1</smiles>

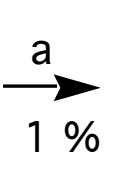

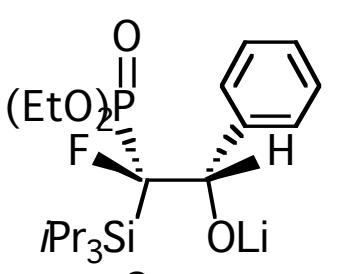<smiles>CCO[P+](=O)C(F)=Cc1ccccc1</smiles>
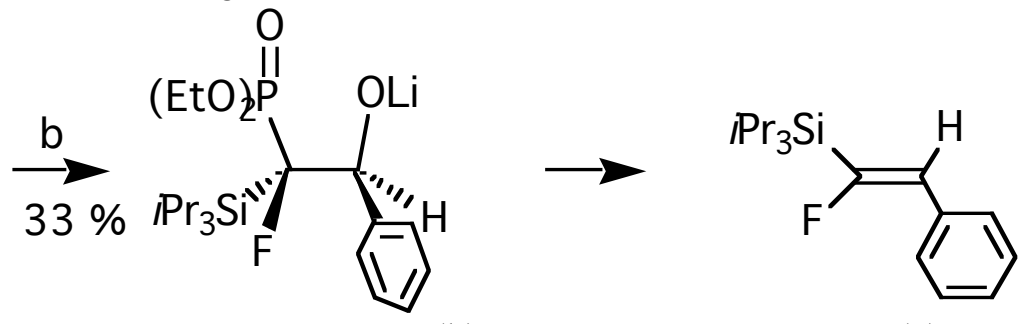

Scheme 6. Competition between Wittig-Horner $(\mathrm{b})$ and Peterson olefination(a) reactions

Secondly, we tried to decrease the $E / Z$ ratio of $6 \mathrm{t}$ (with $Z$ as major isomer) by increasing the size of the trialkylsilane. The effect was not as marked as expected (see Table 2). Only a $3 \%$ decrease was observed using $t$-butyldimethylsilylchloride, one of the largest silyl agents we used. In conclusion when the $E$-isomer is formed as the main product, we find that the bulk of the trialkylsilane group can control the approach of the carbonyl compound. By contrast the bulk of the trialkylsilane group has no influence upon the $E / Z$ ratio when the $Z$ isomer is the main product.

Table 2. Influence of the nature of the chlorotrialkylsilane on the stereoselectivity of the Peterson olefination reaction

\begin{tabular}{lllll}
\hline Carbonyl compound & $\mathrm{R}_{3} \mathrm{SiCl}$ & Yield & $E$ & $Z$ \\
\hline$p-\mathrm{H}_{3} \mathrm{COC}_{6} \mathrm{H}_{4} \mathrm{CHO}$ 6f & $\mathrm{HMe}_{2} \mathrm{SiCl}$ & $90 \%$ & 78 & 22 \\
& $\mathrm{Me}_{3} \mathrm{SiCl}$ & $91 \%$ & 70 & 30 \\
& $t-\mathrm{Bu}(\mathrm{Me})_{2} \mathrm{SiCl}$ & $90 \%$ & 60 & 40 \\
& $\mathrm{Et}_{3} \mathrm{SiCl}$ & $89 \%$ & 56 & 44 \\
& $i-\mathrm{Pr}_{3} \mathrm{SiCl}$ & $50 \%$ & 98 & 2 \\
& & $+33 \% p-\mathrm{H}_{3} \mathrm{COC}_{6} \mathrm{H} 4 \mathrm{CH}=\mathrm{CFSi}(i-\mathrm{Pr})_{3}$ \\
& & $80 \%$ & 24 & 76 \\
\hline
\end{tabular}


The addition of a Lewis base or acid to the reaction medium has no marked effect. For instance reaction of $p$-methoxybenzaldehyde with 2 in the presence of triethylamine (1.5 equiv) did not change the course of the reaction and $\mathbf{6 f}$ was obtained with an $E / Z$ ratio (72/28) equivalent to that obtained without triethylamine (70/30). The addition of lithium bromide (1.5 equiv) to the reaction mixture had a rather negative effect and the reaction gives $6 \mathbf{f}$ contaminated with many by-products containing phosphorus and fluorine.

To conclude this study we wished to evaluate the influence of the fluorine atom on the Peterson olefination reaction against non-fluorinated substrates with regard to steric and electronic effects. Since the double halogen-metal exchange reaction with intermediate monosilylation or the double proton abstraction works well on various substrates giving easy access to $\alpha$-silylated phosphonate derivatives these methods ${ }^{13}$ were performed on diethyl trichloromethylphosphonate, diethyl methylphosphonate, and diethyl ethylphosphonate as starting materials and 2-furaldehyde. Respectively the substrates yielded vinylphosphonates 8, 9, 10 (Table 3). From these results we can see a trend which we attribute to electronic effects on the transition state from the alpha substituent. Electron withdrawing substituents $(\mathrm{X}=\mathrm{F}, \mathrm{Cl})$ appear to be involved in a closed transition state giving one isomer over preference to the other whereas the electron donating substituents $\left(\mathrm{X}=\mathrm{H}, \mathrm{CH}_{3}\right)$ render the transiton state more unstable and prone to conformational change giving a more equal ratio of isomers.

Table 3. Influence of the group in the $\alpha$-position on the stereoselectivity of the Peterson olefination reaction

\begin{tabular}{lllll}
\hline & \\
$\mathrm{X}=\mathrm{F}$ & $\mathbf{6 h}$ & 92 & 80 & 20 \\
$\mathrm{X}=\mathrm{Cl}$ & $\mathbf{8}$ & 89 & $20^{*}$ & $80^{*}$ \\
$\mathrm{X}=\mathrm{H}$ & $\mathbf{9}$ & 78 & 33 & 67 \\
$\mathrm{X}=\mathrm{CH}_{3}$ & $\mathbf{1 0}$ & 93 & 55 & 45 \\
\hline
\end{tabular}

\section{CONCLUSION}

In summary, the simple experimental procedure herein described enables the synthesis of a large variety of $\alpha$-fluorovinylphosphonates in good yield. Also the previously difficult to access $Z$-isomers can now be synthesised in the case where ketones are used as starting materials. This new use of the Peterson olefination reaction should make it an alternative to other methods of $\alpha$-fluorovinylphosphonates synthesis. Furthermore our studies give an insight into the nature of the reaction path with respect to starting materials and experimental conditions which should prove useful in predicting the outcome of the reaction. These $\alpha$-fluorovinylphosphonates are potentially valuable synthetic intermediates for the synthesis of more elaborate $\alpha$-fluoro phosphonates. Work in this laboratory is focusing on further application of this methodology. 


\section{EXPERIMENTAL SECTION}

NMR spectra were recorded in $\mathrm{CDCl}_{3}$ as solvent on a Bruker AC 200 spectrometer operating at 81.01 $\mathrm{MHz}$ for phosphorus, $200 \mathrm{MHz}$ for proton and 50.3 MHz for carbon. ${ }^{31} \mathrm{P}$ downfield shifts $(\delta)$ are expressed with a positive sign, in ppm, relative to external $85 \% \mathrm{H}_{3} \mathrm{PO}_{4}$ in $\mathrm{H}_{2} \mathrm{O} .{ }^{1} \mathrm{H}$ and ${ }^{13} \mathrm{C}$ chemical shifts $(\delta)$ are reported in ppm relative to $\mathrm{CDCl}_{3}$ as internal standard. Positive value of coupling constants $(J)$ are given in Hertz. The following abbreviations are used: s, d, t, q, p, m for singlet, doublet, triplet, quadruplet, pentuplet and multiplet respectively.

Low-resolution mass spectra were recorded on a Hewlet Packard 5989 B mass spectrometer.

Organic solvents were purified by standard procedures. THF was distilled under an inert atmosphere from purple solutions of sodium: benzophenone ketyl. The synthesis of all compounds were carried out under dry nitrogen.

Diethyl 1,1-dibromo-1-fluoromethylphosphonate (1) $\mathrm{C}_{5} \mathrm{H}_{10} \mathrm{O}_{3} \mathrm{PBr}_{2} \mathrm{~F}(\mathrm{M}=328)$ : A $500 \mathrm{~mL}$ flask was charged with tribromofluoromethane $(50 \mathrm{~g}, 0.185 \mathrm{~mol})$ and dry hexane $(100 \mathrm{~mL})$. Triethyl phosphite $(29.9 \mathrm{~g}$, $0.18 \mathrm{~mol}$ ) was then added in one portion. The reaction mixture became turbid and few hours later return clear. The flask was kept in the light for 11-12 $\mathrm{d}$ at $\mathrm{rt}$. The reaction can be monitored by ${ }^{31} \mathrm{P}$ NMR spectroscopy. When all the triethyl phosphite has been consumed, hexane was evaporated under reduced pressure to afford the crude product $(58.5 \mathrm{~g}, 97 \%) . \mathbf{1}$ is distilled by bulb-to-bulb distillation $\left(170-175^{\circ} \mathrm{C} / 20 \mathrm{mmHg}\right)$.

31P NMR +1.6 (d, $\left.{ }^{2} J_{\mathrm{PF}}=77\right) . /{ }^{1} \mathbf{H}$ NMR $1.38\left(\mathrm{t}, 6 \mathrm{H},{ }^{3} J_{\mathrm{HH}}=7.1, \mathrm{CH}_{3} \mathrm{CH}_{2} \mathrm{O}\right), 4.37\left(\mathrm{dqd}, 4 \mathrm{H},{ }^{3} J_{\mathrm{HP}}=8.2,{ }^{3} J_{\mathrm{HH}}=7.1\right.$,

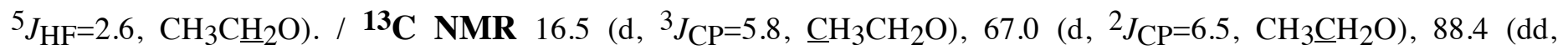
$\left.{ }^{1} J=333.6,{ }^{1} J=202.3, \mathrm{P}(\mathrm{F}) \underline{\mathrm{CFBr}} 2\right) . / \mathbf{~ m} / \mathbf{z}(\mathbf{E I}) 331\left(\mathrm{M}+\mathrm{H}^{+}{ }^{8}{ }^{81} \mathrm{Br}, 49\right), 329\left(\mathrm{M}+\mathrm{H}^{+}{ }^{81} \mathrm{Br}{ }^{79} \mathrm{Br}, 100\right), 327\left(\mathrm{M}+\mathrm{H}^{+}\right.$ $\left.2^{79} \mathrm{Br}, 53\right), 301$ (2 Br, 46), 272 (2 Br, 19), 249-247 (1 Br, 45), 221-219 (1 Br, 68-64).

General procedure for the synthesis of diethyl $\alpha$-fluorovinylphosphonates : A mixture of diethyl 1,1dibromo-1-fluoromethanephosphonate 1 (1.45 g, $4.4 \mathrm{mmol})$ and chlorotrimethylsilane $(0.48 \mathrm{~g}, 4.4 \mathrm{mmol}) \mathrm{in}$ THF $(15 \mathrm{ml})$ were added dropwise to a solution of $n$-BuLi in THF $(15 \mathrm{ml})$ at $-78^{\circ} \mathrm{C}$. The mixture was stirred for $10 \mathrm{~min}$ at this temperature and then a solution of carbonyl compound (4 mmol) in THF (10 ml) was added slowly. After $10 \mathrm{~min}$ stirring at $-78^{\circ} \mathrm{C}$, the reaction mixture was allowed to warm-up to $0^{\circ} \mathrm{C}$ over $30 \mathrm{~min}$. An ice-cold sodium chloride-water solution was then rapidly poured into the reaction mixture. The aqueous layer was extracted with diethyl ether $(3 \times 20 \mathrm{ml})$. The combined organic extracts were dried $\left(\mathrm{MgSO}_{4}\right)$ and evaporated under reduced pressure to afford the crude product which was purified by a column chromatography with silica gel $60(35-70 \mu \mathrm{m}$, SDS ; eluant : hexane / AcOEt : 80 / 20). Usually the Z-isomer is slightly less polar than the $E$-isomer.

Diethyl 1-fluoro-4-phenyl-but-1-enephosphonate 6a : $\mathrm{C}_{14} \mathrm{H}_{20} \mathrm{O}_{3} \mathrm{PF}(\mathrm{M}=286)$

E-isomer: 31P NMR +6.1 (d, $\left.{ }^{2} J_{\mathrm{PF}}=102\right)$. / 31P (1 H coupled) +6.1 (dd, $\left.{ }^{2} J_{\mathrm{PF}}=102,{ }^{2} J_{\mathrm{PHethoxy}}=7\right) . / 1$ H NMR 1.28 $\left(\mathrm{t}, 6 \mathrm{H},{ }^{3} J_{\mathrm{HH}}=7.1, \underline{\mathrm{C}}_{3} \mathrm{CH}_{2} \mathrm{O}\right), 2.55\left(\mathrm{~m}, 2 \mathrm{H}, \mathrm{P}(\mathrm{F}) \mathrm{C}=\mathrm{CHC}_{2}\right), 2.73\left(\mathrm{t}, 2 \mathrm{H},{ }^{3} J_{\mathrm{HH}}=7.1, \mathrm{P}(\mathrm{F}) \mathrm{C}=\mathrm{CHCH}_{2} \mathrm{CH}_{2}\right), 4.03(\mathrm{q}, 4 \mathrm{H}$, $\left.{ }^{3} J_{\mathrm{HH}}=7.1, \mathrm{CH}_{3} \mathrm{C}_{2} \mathrm{O}\right), 5.94\left(\mathrm{dq}, 1 \mathrm{H},{ }^{3} J_{\mathrm{HFtrans}}=39.3,{ }^{3} J_{\mathrm{HPcis}}={ }^{3} J_{\mathrm{HH}}=7.6, \mathrm{P}(\mathrm{F}) \mathrm{C}=\mathrm{C} \underline{\mathrm{H}}\right), 7.2\left(\mathrm{~m}, 5 \mathrm{H}, \mathrm{C}_{6} \underline{\mathrm{H}}_{5}\right) . / \mathbf{1 3}^{\mathrm{C}}$ NMR $16.5\left(\mathrm{~d},{ }^{3} J_{\mathrm{CP}}=6.0, \underline{\mathrm{CH}}_{3} \mathrm{CH}_{2} \mathrm{O}\right), 26.1\left(\mathrm{dd},{ }^{3} J=10.4,{ }^{3} J=4.3, \mathrm{P}(\mathrm{F}) \mathrm{C}=\mathrm{CH}-\underline{\mathrm{CH}}_{2}\right), 34.7\left(\mathrm{~s}, \underline{\mathrm{CH}}_{2} \mathrm{C}_{6} \mathrm{H}_{5}\right), 63.4(\mathrm{~d}$, $\left.{ }^{2} J_{\mathrm{CP}}=5.4, \mathrm{CH}_{3} \underline{\mathrm{CH}} \mathrm{H}_{2} \mathrm{O}\right), 126.0\left(\mathrm{dm},{ }^{2} J=28.1, \mathrm{P}(\mathrm{F}) \mathrm{C}=\underline{\mathrm{CH}}\right), 126.4\left(\mathrm{~s}, \underline{\mathrm{C}}_{\text {para }}\right.$ of $\left.\mathrm{C}_{6} \mathrm{H}_{5}\right), 128.7$ (s, $\underline{\mathrm{C}}_{\text {ortho or meta }}$ of $\left.\mathrm{C}_{6} \mathrm{H}_{5}\right)$, 128.8 (s, $\underline{\mathrm{C}}_{\text {ortho or meta }}$ of $\left.\mathrm{C}_{6} \mathrm{H}_{5}\right), 140.8\left(\mathrm{~s}, \underline{\mathrm{C}}_{\mathrm{ipso}}\right.$ of $\left.\mathrm{C}_{6} \mathrm{H}_{5}\right), 150.4\left(\mathrm{dd},{ }^{1} J=276.2,{ }^{1} J=235.5, \mathrm{P}(\mathrm{F}) \underline{\mathrm{C}}=\mathrm{CH}\right)$. 
Z-isomer: ${ }^{31} \mathbf{P}$ NMR +5.2 (d, $\left.{ }^{2} J_{\mathrm{PF}}=106\right)$. / $31 \mathbf{P}\left(\mathbf{1}_{\mathbf{H}}\right.$ coupled) $\mathbf{N M R}+5.2\left(\mathrm{ddt},{ }^{2} J_{\mathrm{PF}}=106,{ }^{2} J_{\mathrm{PH}}\right.$ trans $=31$, $\left.{ }^{2} J_{\mathrm{PHethoxy}}=6\right) . /{ }^{1} \mathbf{H}$ NMR $1.31\left(\mathrm{t}, 6 \mathrm{H},{ }^{3} \mathrm{~J}_{\mathrm{HH}}=7.1, \mathrm{C}_{3} \mathrm{CH}_{2} \mathrm{O}\right), 2.77\left(\mathrm{~m} 4 \mathrm{H},\left(\mathrm{C}=\mathrm{CHC} \underline{H}_{2}\right)_{2}\right), 4.02(\mathrm{q}, 4 \mathrm{H}$, $\left.{ }^{3} J_{\mathrm{HH}}=7.1, \mathrm{CH}_{3} \mathrm{CH}_{2} \mathrm{O}\right), 6.09\left(\mathrm{ddt}, 1 \mathrm{H},{ }^{3} J_{\mathrm{HFtrans}}=31.6,{ }^{3} J_{\mathrm{HPcis}}=28.6,{ }^{3} J_{\mathrm{HH}}=8.2, \mathrm{P}(\mathrm{F}) \mathrm{C}=\mathrm{C} \underline{\mathrm{H}}\right), 7.23(\mathrm{~m}, 5 \mathrm{H}$,

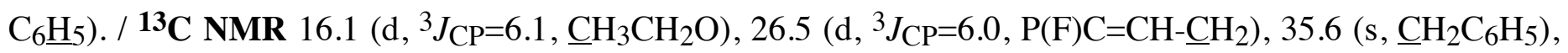
$62.9\left(\mathrm{~d},{ }^{2} J_{\mathrm{CP}}=5.7, \mathrm{CH}_{3} \underline{\mathrm{CH}}_{2} \mathrm{O}\right), 126.1\left(\mathrm{~s}, \underline{\mathrm{C}}_{\mathrm{para}}\right.$ of $\left.\mathrm{C}_{6} \mathrm{H}_{5}\right), 127.3\left(\mathrm{dd},{ }^{2} J=30.7,{ }^{2} J=12.2, \mathrm{P}(\mathrm{F}) \mathrm{C}=\underline{\mathrm{CH}}\right), 128.4(\mathrm{~s}$, $\underline{\mathrm{C}}_{\text {meta }}$ of $\mathrm{C}_{6} \mathrm{H}_{5}$ ), 128.7 (s, $\underline{\mathrm{C}}_{\text {ortho }}$ of $\mathrm{C}_{6} \mathrm{H}_{5}$ ), 140.6 (s, $\underline{\mathrm{C}}_{\text {ipso }}$ of $\mathrm{C}_{6} \mathrm{H}_{5}$ ), 149.6 (dd, ${ }^{1} J=265.3,{ }^{1} J=228.7$, $\mathrm{P}(\mathrm{F}) \underline{\mathrm{C}}=\mathrm{CH}) . /$ m/z (EI) $286\left(\mathrm{M}^{+}, 33\right), 265$ (3), 237 (6), 209 (9).

Diethyl 1-fluoro-3-phenyl-but-1-enephosphonate $6 \mathbf{b}: \mathrm{C}_{14} \mathrm{H}_{20} \mathrm{O}_{3} \mathrm{PF}(\mathrm{M}=286)$

E-isomer: ${ }^{31} \mathbf{P} \mathbf{N M R}+6.1\left(\mathrm{~d},{ }^{2} J_{\mathrm{PF}}=101\right)$. / 31P (1 H coupled) $\mathbf{N M R}+6.1\left(\mathrm{dd},{ }^{2} J_{\mathrm{PF}}=101,{ }^{2} J_{\mathrm{PHethoxy}}=7\right) . / \mathbf{1}_{\mathbf{H}}$ NMR $1.29\left(\mathrm{qd}, 3 \mathrm{H},{ }^{3} J_{\mathrm{HH}}={ }^{5} J_{\mathrm{HPtrans}}={ }^{5} J_{\mathrm{HFcis}}=7.0,{ }^{4} J_{\mathrm{HH}}=0.5, \mathrm{CHC} \underline{H}_{3}\right), 1.38\left(\mathrm{td}, 6 \mathrm{H},{ }^{3} J_{\mathrm{HH}}=6.9,{ }^{4} J_{\mathrm{HP}}=0.5\right.$, $\left.\mathrm{C}_{3} \mathrm{CH}_{2} \mathrm{O}\right), 4.10\left(\mathrm{~m}, 5 \mathrm{H}, \mathrm{CH}_{3} \underline{\mathrm{C}}_{2} \mathrm{O}\right.$ et $\left.\mathrm{P}(\mathrm{F}) \mathrm{C}=\mathrm{CH}-\mathrm{C} \underline{\mathrm{H}}\right), 6.10$ (ddd, $1 \mathrm{H},{ }^{3} J_{\mathrm{HFtrans}}=38.8,{ }^{3} J_{\mathrm{HPcis}}=10.0$,

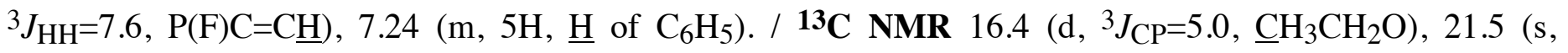
$\left.\mathrm{CH}_{\mathrm{CH}_{3}}\right), 35.2\left(\mathrm{dd},{ }^{3} J=10.0,{ }^{3} J=4.8, \mathrm{P}(\mathrm{F}) \mathrm{C}=\mathrm{CH}-\underline{\mathrm{CH}}\right), 63.3\left(\mathrm{~d},{ }^{2} J_{\mathrm{CP}}=4.1, \mathrm{CH}_{3} \underline{\mathrm{CH}}_{2} \mathrm{O}\right), 127.1$ (s, $\underline{\mathrm{C}}_{\text {ortho }}$ of $\left.\mathrm{C}_{6} \mathrm{H}_{5}\right), 129.0$ (s, $\underline{\mathrm{C}}_{\text {meta }}$ and $\underline{\mathrm{C}}_{\text {para }}$ of $\left.\mathrm{C}_{6} \mathrm{H}_{5}\right), 130.9$ (dd, $\left.{ }^{2} J=27.4,{ }^{2} J=5.8, \mathrm{P}(\mathrm{F}) \mathrm{C}=\underline{\mathrm{CH}}\right), 143.8$ (s, $\underline{\mathrm{C}}_{\text {ipso }}$ of $\left.\mathrm{C}_{6} \mathrm{H}_{5}\right)$, $149.0\left(\mathrm{dd},{ }^{1} J=274.7,{ }^{1} J=235.1, \mathrm{P}(\mathrm{F}) \underline{\mathrm{C}}=\mathrm{CH}\right) . / \mathbf{~ m} / \mathbf{z}(\mathbf{E I}) 287\left(\mathrm{M}+\mathrm{H}^{+}, 52\right), 266(27), 210$ (26), 129 (100).

Z-isomer: ${ }^{31} \mathbf{P} \mathbf{N M R}+4.5\left(\mathrm{~d},{ }^{2} J_{\mathrm{PF}}=104\right)$. / $31 \mathbf{P}\left({ }^{1} \mathbf{H}\right.$ coupled) $\mathbf{N M R}+4.5\left(\mathrm{ddt},{ }^{2} J_{\mathrm{PF}}=104,{ }^{2} J_{\mathrm{PHtrans}}=31\right.$, $\left.{ }^{2} J_{\mathrm{PHethoxy}}=6\right) . /{ }^{1} \mathbf{H}$ NMR $1.27\left(\mathrm{t}, 3 \mathrm{H},{ }^{3} \mathrm{~J}_{\mathrm{HH}}=7.0, \mathrm{C}_{3} \mathrm{CH}_{2} \mathrm{O}\right), 1.36\left(\mathrm{t}, 3 \mathrm{H},{ }^{3} \mathrm{~J}_{\mathrm{HH}}=7.2, \mathrm{C}_{3} \mathrm{CH}_{2} \mathrm{O}\right), 1.39$ (dd, $\left.3 \mathrm{H},{ }^{3} \mathrm{~J}_{\mathrm{HH}}=6.9,{ }^{4} \mathrm{~J}_{\mathrm{HH}}=0.9, \mathrm{CHC}_{3}\right), 4.12\left(\mathrm{~m}, 4 \mathrm{H}, \mathrm{CH}_{3} \underline{\mathrm{H}}_{2} \mathrm{O}\right), 4.54(\mathrm{~m}, 1 \mathrm{H}, \mathrm{P}(\mathrm{F}) \mathrm{C}=\mathrm{CH}-\mathrm{C} \underline{\mathrm{H}}), 6.20(\mathrm{ddd}, 1 \mathrm{H}$, $\left.{ }^{3} J_{\mathrm{HFtrans}}=31.3,{ }^{3} J_{\mathrm{HPcis}}=28.1,{ }^{3} J_{\mathrm{HH}}=11.5, \mathrm{P}(\mathrm{F}) \mathrm{C}=\mathrm{C} \underline{\mathrm{H}}\right), 7.27\left(\mathrm{~m}, 5 \mathrm{H}, \underline{\mathrm{H}}\right.$ of $\left.\mathrm{C}_{6} \mathrm{H}_{5}\right) . / \mathbf{1 3}^{\mathbf{C}} \mathbf{N M R} 16.3(\mathrm{~d}$,

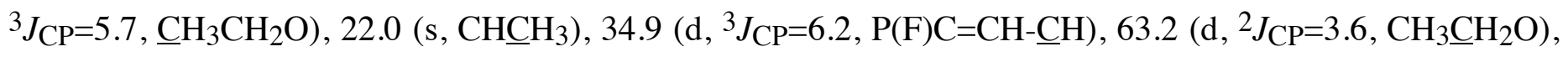
126.8 (s, $\underline{\mathrm{C}}_{\text {para }}$ of $\mathrm{C}_{6} \mathrm{H}_{5}$ ), 127.0 (s, $\underline{\mathrm{C}}_{\text {ortho }}$ of $\mathrm{C}_{6} \mathrm{H}_{5}$ ), 128.8 (s, $\underline{\mathrm{C}}_{\text {meta }}$ of $\mathrm{C}_{6} \mathrm{H}_{5}$ ), 132.8 (dd, ${ }^{2} J=31.3,{ }^{2} J=11.2$, $\mathrm{P}(\mathrm{F}) \mathrm{C}=\underline{\mathrm{CH}}), 144.5\left(\mathrm{~s}, \underline{\mathrm{C}}_{\mathrm{ipso}}\right.$ of $\left.\mathrm{C}_{6} \mathrm{H}_{5}\right), 148.5\left(\mathrm{dd},{ }^{1} \mathrm{~J}=266.8,{ }^{1} \mathrm{~J}=228.6, \mathrm{P}(\mathrm{F}) \underline{\mathrm{C}}=\mathrm{CH}\right) . / \mathbf{m} / \mathbf{z}(\mathbf{E I}) 287\left(\mathrm{M}+\mathrm{H}^{+}\right.$, 27), 266 (17), 210 (28), 129 (61).

Diethyl 1-fluoro -3-methylpent-1-enephosphonate $\mathbf{6 c}: \mathrm{C}_{10} \mathrm{H}_{20} \mathrm{O}_{3} \mathrm{PF}(\mathrm{M}=238)$

E-isomer: ${ }^{31} \mathbf{P}$ NMR +6.2 (d, $\left.{ }^{2} J_{\mathrm{PF}}=103\right)$. / $1 \mathbf{H}$ NMR $0.84\left(\mathrm{t}, 3 \mathrm{H},{ }^{3} \mathrm{~J}_{\mathrm{HH}}=7.3, \mathrm{CH}_{2} \mathrm{CH}_{3}\right), 1.00\left(\mathrm{~d}, 3 \mathrm{H},{ }^{3} \mathrm{~J}_{\mathrm{HH}}=6.7\right.$, $\left.\mathrm{CHC}_{3}\right), 1.31\left(\mathrm{~m}, 2 \mathrm{H}, \underline{\mathrm{C}}_{2} \mathrm{CH}_{3}\right), 1.32\left(\mathrm{t}, 6 \mathrm{H},{ }^{3} \mathrm{~J}_{\mathrm{HH}}=7.1, \underline{\mathrm{CH}}_{3} \mathrm{CH}_{2} \mathrm{O}\right), 2.67(\mathrm{~m}, 1 \mathrm{H}, \mathrm{P}(\mathrm{F}) \mathrm{C}=\mathrm{CH}-\mathrm{C} \underline{\mathrm{H}}), 4.15(\mathrm{~m}$, $\left.4 \mathrm{H}, \mathrm{CH}_{3} \underline{\mathrm{C}}_{2} \mathrm{O}\right), 5.74\left(\mathrm{ddd}, 1 \mathrm{H},{ }^{3} J_{\mathrm{HFtrans}}=40.1,{ }^{3} J_{\mathrm{HPcis}}=10.1,{ }^{3} J_{\mathrm{HH}}=7.8, \mathrm{P}(\mathrm{F}) \mathrm{C}=\mathrm{C} \underline{\mathrm{H}}\right) . /{ }^{\mathbf{1 3}} \mathbf{C} \mathbf{~ N M R} 12.0(\mathrm{~s}$, $\left.\mathrm{CH}_{2} \underline{\mathrm{CH}}_{3}\right), 16.4\left(\mathrm{~d},{ }^{3} J_{\mathrm{CP}}=6.1, \underline{\mathrm{CH}}_{3} \mathrm{CH}_{2} \mathrm{O}\right), 20.1\left(\mathrm{~s}, \mathrm{CHCH}_{3}\right), 29.5\left(\mathrm{~s}, \underline{\mathrm{CH}}_{2} \mathrm{CH}_{3}\right), 31.6\left(\mathrm{dd},{ }^{3} J=10.3,{ }^{3} J=2.8\right.$, $\mathrm{P}(\mathrm{F}) \mathrm{C}=\mathrm{CH}-\underline{\mathrm{CH}}), 63.1\left(\mathrm{~d},{ }^{2} J_{\mathrm{CP}}=3.6, \mathrm{CH}_{3} \underline{\mathrm{CH}}_{2} \mathrm{O}\right), 132.3\left(\mathrm{dd},{ }^{2} J=26.9,{ }^{2} J=5.4, \mathrm{P}(\mathrm{F}) \mathrm{C}=\underline{\mathrm{CH}}\right), 149.2\left(\mathrm{dd},{ }^{1} J=273.0\right.$, $\left.{ }^{1} J=235.1, \mathrm{P}(\mathrm{F}) \underline{\mathrm{C}}=\mathrm{CH}\right)$.

Z-isomer: ${ }^{31} \mathbf{P} \mathbf{N M R}+4.8\left(\mathrm{~d},{ }^{2} J_{\mathrm{PF}}=105\right)$. / 31P $\left(\mathbf{1}_{\mathbf{H}}\right.$ coupled) $\mathbf{N M R}+4.8\left(\mathrm{ddd},{ }^{2} J_{\mathrm{PF}}=105,{ }^{2} J_{\mathrm{PHtrans}}=31\right.$, $\left.{ }^{2} J_{\mathrm{PHethoxy}}=5\right)$. / ${ }^{\mathbf{1}} \mathbf{H}$ NMR $0.87\left(\mathrm{t}, 3 \mathrm{H},{ }^{3} J_{\mathrm{HH}}=7.4, \mathrm{CH}_{2} \underline{\mathrm{H}}_{3}\right), 0.99\left(\mathrm{dd}, 3 \mathrm{H},{ }^{3} J_{\mathrm{HH}}=6.7,{ }^{4} J_{\mathrm{HH}}=1.0, \mathrm{CHC}_{3}\right)$, $1.31\left(\mathrm{~m}, 2 \mathrm{H}, \mathrm{CH}_{2} \mathrm{CH}_{3}\right), 1.34\left(\mathrm{t}, 6 \mathrm{H},{ }^{3} \mathrm{~J}_{\mathrm{HH}}=7.1, \mathrm{C}_{3} \mathrm{CH}_{2} \mathrm{O}\right), 3.04\left(\mathrm{~m}, 1 \mathrm{H}, \mathrm{C} \underline{\mathrm{H}}\left(\mathrm{CH}_{3}\right) \mathrm{C}_{2} \mathrm{H}_{5}\right), 4.14(\mathrm{~m}, 4 \mathrm{H}$, $\left.{ }^{3} J_{\mathrm{HH}}=7.1,{ }^{3} J_{\mathrm{HP}}=2.7, \mathrm{CH}_{3} \underline{\mathrm{H}}_{2} \mathrm{O}\right), 5.84\left(\mathrm{ddd}, 1 \mathrm{H},{ }^{3} J_{\mathrm{HPtrans}}=31.7,{ }^{3} J_{\mathrm{HFcis}}=29.2,{ }^{3} J_{\mathrm{HH}}=11.4, \mathrm{P}(\mathrm{F}) \mathrm{C}=\mathrm{C} \underline{\mathrm{H}}\right) . /{ }^{13} \mathrm{C}$ NMR $11.9\left(\mathrm{~s}, \mathrm{CH}_{2} \underline{\mathrm{CH}}_{3}\right), 16.4\left(\mathrm{~d},{ }^{3} \mathrm{~J}_{\mathrm{CP}}=6.2, \underline{\mathrm{CH}}_{3} \mathrm{CH}_{2} \mathrm{O}\right), 21.3\left(\mathrm{~s}, \mathrm{CHCH}_{3}\right), 30.3\left(\mathrm{~s}, \underline{\mathrm{CH}}_{2} \mathrm{CH}_{3}\right), 31.6\left(\mathrm{~d},{ }^{3} \mathrm{~J}=5.8\right.$, $\mathrm{P}(\mathrm{F}) \mathrm{C}=\mathrm{CH}-\underline{\mathrm{CH}}), 63.0\left(\mathrm{~d},{ }^{2} J_{\mathrm{CP}}=5.2, \mathrm{CH}_{3} \underline{\mathrm{CH}}_{2} \mathrm{O}\right), 133.7\left(\mathrm{dd},{ }^{2} J=31.8,{ }^{2} J=9.4, \mathrm{P}(\mathrm{F}) \mathrm{C}=\underline{\mathrm{CH}}\right), 148.9\left(\mathrm{dd},{ }^{1} J=264.1\right.$, $\left.{ }^{1} J=229.0, \mathrm{P}(\mathrm{F}) \underline{\mathrm{C}}=\mathrm{CH}\right)$. 
Diethyl 1-fluoro-3,3-dimethylbut-1-enephosphonate $\mathbf{6 d}$ : $\mathrm{C}_{10} \mathrm{H}_{20} \mathrm{O}_{3} \mathrm{PF}(\mathrm{M}=238)$

E-isomer: ${ }^{31} \mathbf{P} \mathbf{N M R}+6.5\left(\mathrm{~d},{ }^{2} J_{\mathrm{PF}}=104\right) . /{ }^{1} \mathbf{H} \mathbf{N M R} 1.17\left(\mathrm{~d}, 9 \mathrm{H},{ }^{4} J_{\mathrm{HH}}=1.0, \mathrm{C}\left(\mathrm{C}_{3}\right)_{3}\right), 1.34\left(\mathrm{t}, 6 \mathrm{H},{ }^{3} J_{\mathrm{HH}}=7.0\right.$, $\left.\mathrm{C}_{3} \mathrm{CH}_{2} \mathrm{O}\right), 4.13\left(\mathrm{dqd}, 4 \mathrm{H},{ }^{3} J_{\mathrm{HP}}=8.4,{ }^{3} J_{\mathrm{HH}}=7.0,{ }^{5} J_{\mathrm{HF}}=1.5, \mathrm{CH}_{3} \underline{C H}_{2} \mathrm{O}\right), 5.85$ (dd, $1 \mathrm{H},{ }^{3} J_{\mathrm{HFtrans}}=46.2$, $\left.{ }^{3} J_{\mathrm{HPcis}}=9.1, \mathrm{P}(\mathrm{F}) \mathrm{C}=\mathrm{C} \underline{\mathrm{H}}\right) . /{ }^{13} \mathrm{C}$ NMR $16.6\left(\mathrm{~d},{ }^{3} J_{\mathrm{CP}}=6.1, \underline{\mathrm{CH}}_{3} \mathrm{CH}_{2} \mathrm{O}\right), 30.2\left(\mathrm{~d},{ }^{4} J=2.1, \mathrm{C}\left(\underline{\mathrm{CH}_{3}}\right)_{3}\right), 33.4(\mathrm{~d}$, $\left.{ }^{3} J=11.7, \underline{\mathrm{C}}\left(\mathrm{CH}_{3}\right)_{3}\right), 63.2\left(\mathrm{~d},{ }^{2} J_{\mathrm{CP}}=5.2, \mathrm{CH}_{3} \underline{\mathrm{C}} \mathrm{H}_{2} \mathrm{O}\right), 135.2\left(\mathrm{~d},{ }^{2} J=26.3, \mathrm{P}(\mathrm{F}) \mathrm{C}=\underline{\mathrm{CH}}\right), 149.0\left(\mathrm{dd},{ }^{1} J=277.7\right.$, $\left.{ }^{1} J=236.4, \mathrm{P}(\mathrm{F}) \underline{\mathrm{C}}\right) . / \mathbf{~ m} / \mathbf{z}(\mathbf{E I}) 238\left(\mathrm{M}^{+}, 5 \%\right), 218(15), 190(24), 167$ (68).

Z-isomer: ${ }^{31} \mathbf{P} \mathbf{N M R}+4.0\left(\mathrm{~d},{ }^{2} J_{\mathrm{PF}}=111\right)$. / $\mathbf{1}_{\mathbf{H}} \mathbf{N M R} 1.24\left(\mathrm{~s}, 9 \mathrm{H}, \mathrm{C}\left(\mathrm{CH}_{3}\right)_{3}\right), 1.34\left(\mathrm{t}, 6 \mathrm{H},{ }^{3} J_{\mathrm{HH}}=7.0\right.$, $\left.\mathrm{C}_{3} \mathrm{CH}_{2} \mathrm{O}\right), 4.13\left(\mathrm{~m}, 4 \mathrm{H}, \mathrm{CH}_{3} \underline{\mathrm{H}}_{2} \mathrm{O}\right), 6.21\left(\mathrm{dd}, 1 \mathrm{H},{ }^{3} J_{\mathrm{HPtrans}}=37.8,{ }^{3} J_{\mathrm{HFcis}}=31.7, \mathrm{P}(\mathrm{F}) \mathrm{C}=\mathrm{C} \underline{\mathrm{H}}\right) . /{ }^{13} \mathrm{C}$ NMR

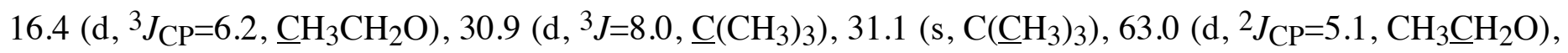
$138.5\left(\mathrm{dd},{ }^{2} J=30.3,{ }^{2} J=13.6, \mathrm{P}(\mathrm{F}) \mathrm{C}=\underline{\mathrm{C}} \mathrm{H}\right), 149.5\left(\mathrm{dd},{ }^{1} J=258.2,{ }^{1} J=227.6, \mathrm{P}(\mathrm{F}) \underline{\mathrm{C}}\right)$.

Diethyl 1-fluoro-2-phenylethenephosphonate $6 \mathbf{e}: \mathrm{C}_{12} \mathrm{H}_{16} \mathrm{O}_{3} \mathrm{PF}(\mathrm{M}=258)$

E-isomer: ${ }^{31} \mathbf{P} \mathbf{N M R}+6.0\left(\mathrm{~d},{ }^{2} J_{\mathrm{PF}}=98\right)$. / ${ }^{1} \mathbf{H}$ NMR $1.37\left(\mathrm{t}, 6 \mathrm{H},{ }^{3} J_{\mathrm{HH}}=7.1, \mathrm{C}_{3} \mathrm{CH}_{2} \mathrm{O}\right), 4.19(\mathrm{dqd}, 4 \mathrm{H}$, $\left.{ }^{3} J_{\mathrm{HP}}=8.1,{ }^{3} J_{\mathrm{HH}}=7.1,{ }^{3} J_{\mathrm{HF}}=2.1, \mathrm{CH}_{3} \underline{\mathrm{H}}_{2} \mathrm{O}\right), 6.72\left(\mathrm{dd}, 1 \mathrm{H},{ }^{3} J_{\mathrm{HFtrans}}=42.3,{ }^{3} J_{\mathrm{HPcis}}=8.6, \mathrm{P}(\mathrm{F}) \mathrm{C}=\mathrm{CH}\right), 7.36(\mathrm{~m}$, $3 \mathrm{H},{ }^{3} J_{\mathrm{HH}}=8.8, \underline{\mathrm{H}}_{\text {meta }}$ and $\underline{\mathrm{H}}_{\text {para }}$ of $\left.\mathrm{C}_{6} \mathrm{H}_{5}\right), 7.60\left(\mathrm{~m}, 2 \mathrm{H}, \underline{\mathrm{H}}_{\text {ortho }}\right.$ of $\left.\mathrm{C}_{6} \mathrm{H}_{5}\right) . /{ }^{13} \mathrm{C}$ NMR $16.5\left(\mathrm{~d},{ }^{3} J_{\mathrm{CP}}=6.2\right.$,

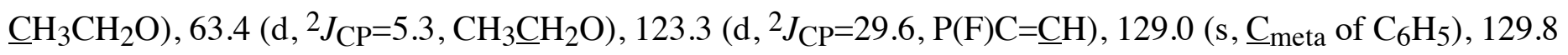
(s, $\underline{\mathrm{C}}_{\text {para }}$ of $\left.\mathrm{C}_{6} \mathrm{H}_{5}\right), 130.3\left(\mathrm{~d},{ }^{4} J_{\mathrm{CP}}=7.6, \underline{\mathrm{C}}_{\text {ortho }}\right.$ of $\left.\mathrm{C}_{6} \mathrm{H}_{5}\right), 131.4\left(\mathrm{~d},{ }^{3} J_{\mathrm{CP}}=14.3, \underline{\mathrm{C}}_{\text {ipso }}\right.$ of $\left.\mathrm{C}_{6} \mathrm{H}_{5}\right), 152.8$ (dd, $\left.{ }^{1} J=286.1,{ }^{1} J=236.0, \mathrm{P}(\mathrm{F}) \underline{\mathrm{C}}=\mathrm{CH}\right)$.

Z-isomer: ${ }^{31} \mathbf{P} \mathbf{N M R}+3.7\left(\mathrm{~d},{ }^{2} J_{\mathrm{PF}}=110\right)$. / $\mathbf{1}_{\mathbf{H}} \mathbf{N M R} 1.21\left(\mathrm{t}, 6 \mathrm{H},{ }^{3} \mathrm{~J}_{\mathrm{HH}}=7.1, \mathrm{C}_{3} \mathrm{CH}_{2} \mathrm{O}\right), 4.08(\mathrm{~m}, 4 \mathrm{H}$, $\left.\mathrm{CH}_{3} \underline{\mathrm{C}}_{2} \mathrm{O}\right), 7.09\left(\mathrm{t}, 1 \mathrm{H},{ }^{3} \mathrm{~J}_{\mathrm{HH}}=29.2, \mathrm{P}(\mathrm{F}) \mathrm{C}=\mathrm{C} \underline{\mathrm{H}}\right), 7.34\left(\mathrm{~m}, 3 \mathrm{H}, \underline{\mathrm{H}}_{\text {meta }}\right.$ and $\underline{\mathrm{H}}_{\text {para }}$ de $\left.\mathrm{C}_{6} \mathrm{H}_{5}\right), 7.49\left(\mathrm{~m}, 2 \mathrm{H}, \underline{\mathrm{H}}_{\text {ortho }}\right.$ of $\left.\mathrm{C}_{6} \mathrm{H}_{5}\right) . /{ }^{13} \mathrm{C}$ NMR $16.2\left(\mathrm{~d},{ }^{3} J_{\mathrm{CP}}=6.2, \underline{\mathrm{CH}}_{3} \mathrm{CH}_{2} \mathrm{O}\right), 63.5\left(\mathrm{~d},{ }^{2} J_{\mathrm{CP}}=6.0, \mathrm{CH}_{3} \underline{\mathrm{CH}}_{2} \mathrm{O}\right), 125.9\left(\mathrm{dd},{ }^{2} J_{\mathrm{CP}}=27.5\right.$, 21.1, $\mathrm{P}(\mathrm{F}) \mathrm{C}=\underline{\mathrm{CH}}), 128.4\left(\mathrm{~s}, \underline{\mathrm{C}}_{\text {meta }}\right.$ of $\left.\mathrm{C}_{6} \mathrm{H}_{5}\right), 129.0\left(\mathrm{~s}, \underline{\mathrm{C}}_{\text {para }}\right.$ of $\left.\mathrm{C}_{6} \mathrm{H}_{5}\right), 129.8$ ( $\mathrm{s}_{\text {broad }}, \underline{\mathrm{C}}_{\text {ortho }}$ of $\left.\mathrm{C}_{6} \mathrm{H}_{5}\right), 130.6(\mathrm{~d}$, ${ }^{3} J=13.1, \underline{\mathrm{C}}_{\text {ipso }}$ of $\left.\mathrm{C}_{6} \mathrm{H}_{5}\right), 151.1\left(\mathrm{dd},{ }^{1} J=267.2,{ }^{1} \mathrm{~J}=230.1, \mathrm{P}(\mathrm{F}) \underline{\mathrm{C}}=\mathrm{CH}\right)$.

Diethyl 1-fluoro 2-(4'-methoxy)phenylethenephosphonate $6 \mathbf{f}: \mathrm{C}_{13} \mathrm{H}_{18} \mathrm{O}_{4} \mathrm{PF}(\mathrm{M}=288)$

E-isomer: ${ }^{31} \mathbf{P} \mathbf{N M R}+6.6\left(\mathrm{~d},{ }^{2} J_{\mathrm{PF}}=98\right)$. / 1 H NMR $1.37\left(\mathrm{t}, 6 \mathrm{H},{ }^{3} J_{\mathrm{HH}}=7.1, \mathrm{C}_{3} \mathrm{CH}_{2} \mathrm{O}\right), 3.81\left(\mathrm{~s}, 3 \mathrm{H}, \mathrm{OC}_{3}\right)$, $4.19\left(\mathrm{pd}, 4 \mathrm{H},{ }^{3} J_{\mathrm{HP}}=8.0, \mathrm{CH}_{3} \underline{\mathrm{H}}_{2} \mathrm{O}\right), 6.68\left(\mathrm{dd}, 1 \mathrm{H},{ }^{3} J_{\mathrm{HFtrans}}=42.8,{ }^{3} J_{\mathrm{HPcis}}=8.5, \mathrm{P}(\mathrm{F}) \mathrm{C}=\mathrm{CH}\right), 6.89(\mathrm{~d}, 2 \mathrm{H}$, ${ }^{3} J_{\mathrm{HH}}=8.8, \underline{\mathrm{H}}_{\text {meta }}$ of $\left.\mathrm{C}_{6} \mathrm{H}_{4}\right), 7.56\left(\mathrm{~d}, 2 \mathrm{H},{ }^{3} J_{\mathrm{HH}}=8.8, \underline{\mathrm{H}}_{\text {ortho }}\right.$ of $\left.\mathrm{C}_{6} \mathrm{H}_{4}\right)$. / ${ }^{13} \mathrm{C}$ NMR $16.5\left(\mathrm{~d},{ }^{3} J_{\mathrm{CP}}=6.0\right.$, $\left.\mathrm{CH}_{3} \mathrm{CH}_{2} \mathrm{O}\right), 55.5\left(\mathrm{~s}, 0 \mathrm{CH}_{3}\right), 63.5\left(\mathrm{~d},{ }^{2} J_{\mathrm{CP}}=5.1, \mathrm{CH}_{3} \underline{\mathrm{CH}}_{2} \mathrm{O}\right), 114.4\left(\mathrm{~s}, \underline{\mathrm{C}}_{\text {meta }}\right.$ of $\left.\mathrm{C}_{6} \mathrm{H}_{4}\right), 123.2\left(\mathrm{~d},{ }^{3} J_{\mathrm{CP}}=30.5\right.$, $\underline{\mathrm{C}}_{\text {ipso }}$ of $\left.\mathrm{C}_{6} \mathrm{H}_{4}\right), 125.2\left(\mathrm{~d},{ }^{2} J_{\mathrm{CP}}=15.8, \mathrm{P}(\mathrm{F}) \mathrm{C}=\underline{\mathrm{C}} \mathrm{H}\right), 132.0\left(\mathrm{~d},{ }^{4} J_{\mathrm{CP}}=7.7, \underline{\mathrm{C}}_{\text {ortho }}\right.$ of $\left.\mathrm{C}_{6} \mathrm{H}_{4}\right), 148.5\left(\mathrm{dd},{ }^{1} \mathrm{~J}=281.7\right.$, $\left.{ }^{1} J=238.9, \mathrm{P}(\mathrm{F}) \underline{\mathrm{C}}=\mathrm{CH}\right), 160.8\left(\mathrm{~d},{ }^{6} J=2.8, \underline{\mathrm{C}}_{\text {para }}\right.$ of $\left.\mathrm{C}_{6} \mathrm{H}_{4}\right) . / \mathbf{~ m} / \mathbf{z}(\mathbf{E I}) 288\left(\mathrm{M}^{+}, 53\right), 240(23), 225(25), 215$ (16).

Z-isomer: ${ }^{31} \mathbf{P} \mathbf{N M R}+4.3\left(\mathrm{~d},{ }^{2} J_{\mathrm{PF}}=110\right)$. / ${ }^{\mathbf{1}} \mathbf{H} \mathbf{N M R} 1.25\left(\mathrm{t}, 6 \mathrm{H},{ }^{3} \mathrm{~J}_{\mathrm{HH}}=7.1, \mathrm{CH}_{3} \mathrm{CH}_{2} \mathrm{O}\right), 3.79\left(\mathrm{~s}, 3 \mathrm{H}, \mathrm{OCH}_{3}\right)$, $4.09\left(\mathrm{~m}, 4 \mathrm{H}, \mathrm{CH}_{3} \underline{\mathrm{CH}}_{2} \mathrm{O}\right), 6.9\left(\mathrm{~m}, 3 \mathrm{H}, \mathrm{P}(\mathrm{F}) \mathrm{C}=\mathrm{C} \underline{\mathrm{H}}\right.$ and $\underline{\mathrm{H}}_{\text {meta }}$ of $\left.\mathrm{C}_{6} \mathrm{H}_{4}\right), 7.47\left(\mathrm{~d}, 2 \mathrm{H},{ }^{3} J_{\mathrm{HH}}=8.6, \underline{\mathrm{H}}_{\mathrm{ortho}}\right.$ of

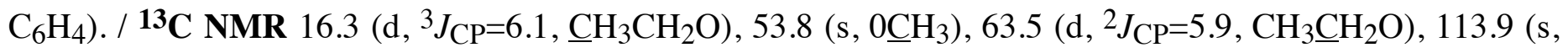
$\underline{\mathrm{C}}_{\text {meta }}$ of $\left.\mathrm{C}_{6} \mathrm{H}_{4}\right), 122.5$ (d, $\underline{\mathrm{C}}_{\text {ipso }}$ of $\left.\mathrm{C}_{6} \mathrm{H}_{4}\right), 125.9\left(\mathrm{dd},{ }^{2} J_{\mathrm{CP}}=21.4,5.7, \mathrm{P}(\mathrm{F}) \mathrm{C}=\underline{\mathrm{C}} \mathrm{H}\right), 131.0$ (s, $\underline{\mathrm{C}}_{\text {ortho }}$ of $\left.\mathrm{C}_{6} \mathrm{H}_{4}\right)$, $149.7\left(\mathrm{dd},{ }^{1} \mathrm{~J}=263.9,{ }^{1} \mathrm{~J}=230.5, \mathrm{P}(\mathrm{F}) \underline{\mathrm{C}}=\mathrm{CH}\right), 160.4\left(\mathrm{~s}, \underline{\mathrm{C}}_{\text {para }}\right.$ of $\left.\mathrm{C}_{6} \mathrm{H}_{4}\right) . / \mathbf{~ m} / \mathbf{z}(\mathbf{E I}) 288\left(\mathrm{M}^{+}, 21\right), 240(13), 225$ (18), 215 (11). 
Diethyl 1-fluoro-2-(4'-dimethylamino)phenylethenephosphonate $\mathbf{6 g}: \mathrm{C}_{14} \mathrm{H}_{21} \mathrm{O}_{3} \mathrm{NPF}(\mathrm{M}=301)$

E-isomer: ${ }^{31} \mathbf{P} \mathbf{N M R}+7.8\left(\mathrm{~d},{ }^{2} J_{\mathrm{PF}}=98\right)$. / ${ }^{1} \mathbf{H}$ NMR $1.36\left(\mathrm{t}, 6 \mathrm{H},{ }^{3} \mathrm{~J}_{\mathrm{HH}}=7.1, \mathrm{C}_{3} \mathrm{CH}_{2} \mathrm{O}\right), 2.99\left(\mathrm{~s}, 6 \mathrm{H}, \mathrm{N}\left(\mathrm{C} \underline{H}_{3}\right)_{2}\right)$, $4.18\left(\mathrm{pd}, 4 \mathrm{H},{ }^{3} J_{\mathrm{HP}}={ }^{3} J_{\mathrm{HH}}=7.1, J=2.0, \mathrm{CH}_{3} \underline{\mathrm{H}}_{2} \mathrm{O}\right), 6.65\left(\mathrm{dd}, 1 \mathrm{H},{ }^{3} J_{\mathrm{HFtrans}}=43.7,{ }^{3} J_{\mathrm{HPcis}}=8.4, \mathrm{P}(\mathrm{F}) \mathrm{C}=\mathrm{C} \underline{\mathrm{H}}\right)$, $6.66\left(\mathrm{~d}, 2 \mathrm{H},{ }^{3} \mathrm{~J}_{\mathrm{HH}}=8.9, \underline{\mathrm{H}}_{\text {meta }}\right.$ of $\left.\mathrm{C}_{6} \mathrm{H}_{4}\right), 7.51\left(\mathrm{~d}, 2 \mathrm{H},{ }^{3} \mathrm{~J}_{\mathrm{HH}}=8.9, \underline{\mathrm{H}}_{\text {ortho }}\right.$ of $\left.\mathrm{C}_{6} \mathrm{H}_{4}\right) . /{ }^{13} \mathrm{C}$ NMR $15.9(\mathrm{~d}$, $\left.{ }^{3} J_{\mathrm{CP}}=6.2, \underline{\mathrm{CH}}_{3} \mathrm{CH}_{2} \mathrm{O}\right), 39.5\left(\mathrm{~s}, \mathrm{~N}\left(\underline{\mathrm{CH}}_{3}\right)_{2}\right), 62.5\left(\mathrm{~d},{ }^{2} J_{\mathrm{CP}}=5.8, \mathrm{CH}_{3} \underline{\mathrm{CH}}_{2} \mathrm{O}\right), 111.4\left(\mathrm{~s}, \underline{\mathrm{C}}_{\text {meta }}\right.$ of $\left.\mathrm{C}_{6} \mathrm{H}_{4}\right), 123.6(\mathrm{~d}$, ${ }^{3} J_{\mathrm{CP}}=30.6, \underline{\mathrm{C}}_{\mathrm{ipso}}$ of $\left.\mathrm{C}_{6} \mathrm{H}_{4}\right), 126.7\left(\mathrm{dd},{ }^{2} J_{\mathrm{CP}}=28.7,21.3, \mathrm{P}(\mathrm{F}) \mathrm{C}=\underline{\mathrm{CH}}\right), 131.2\left(\mathrm{~d},{ }^{4} J_{\mathrm{CP}}=7.5, \underline{\mathrm{C}}_{\text {ortho }}\right.$ of $\left.\mathrm{C}_{6} \mathrm{H}_{4}\right)$, $146.2\left(\mathrm{dd},{ }^{1} \mathrm{~J}=277.7,{ }^{1} \mathrm{~J}=240.8, \mathrm{P}(\mathrm{F}) \underline{\mathrm{C}}=\mathrm{CH}\right), 150.6\left(\mathrm{~d},{ }^{6} \mathrm{~J}=2.1, \underline{\mathrm{C}}_{\mathrm{para}}\right.$ of $\left.\mathrm{C}_{6} \mathrm{H}_{4}\right)$.

Z-isomer: 31P NMR +5.6 (d, $\left.{ }^{2} J_{\mathrm{PF}}=109\right)$. / $1 \mathbf{H} \mathbf{~ N M R ~} 1.27\left(\mathrm{t}, 6 \mathrm{H},{ }^{3} J_{\mathrm{HH}}=7.1, \mathrm{CH}_{3} \mathrm{CH}_{2} \mathrm{O}\right), 2.96(\mathrm{~s}, 6 \mathrm{H}$, $\left.\mathrm{N}\left(\mathrm{CH}_{3}\right)_{2}\right), 4.10\left(\mathrm{~m}, 4 \mathrm{H}, \mathrm{CH}_{3} \underline{\mathrm{H}}_{2} \mathrm{O}\right), 6.65\left(\mathrm{~d}, 2 \mathrm{H},{ }^{3} \mathrm{~J}_{\mathrm{HH}}=8.8, \underline{\mathrm{H}}_{\text {meta }}\right.$ of $\left.\mathrm{C}_{6} \mathrm{H}_{4}\right), 7.02$ (dd, $1 \mathrm{H},{ }^{3} J=31.7$, $\left.{ }^{3} J_{\mathrm{H}}=29.4, \mathrm{P}(\mathrm{F}) \mathrm{C}=\mathrm{C} \underline{\mathrm{H}}\right), 7.51\left(\mathrm{~d}, 2 \mathrm{H},{ }^{3} J_{\mathrm{HH}}=8.8, \underline{\mathrm{H}}_{\mathrm{ortho}}\right.$ of $\left.\mathrm{C}_{6} \mathrm{H}_{4}\right) . /{ }^{13} \mathbf{C} \mathbf{N M R} 15.8\left(\mathrm{~d},{ }^{3} J_{\mathrm{CP}}=8.8, \underline{\mathrm{CH}}_{3} \mathrm{CH}_{2} \mathrm{O}\right)$, $39.6\left(\mathrm{~s}, \mathrm{~N}\left(\underline{\mathrm{CH}}_{3}\right)_{2}\right), 62.6\left(\mathrm{~d},{ }^{2} J_{\mathrm{CP}}=7.2, \mathrm{CH}_{3} \underline{\mathrm{CH}}_{2} \mathrm{O}\right), 111.2$ (s, $\underline{\mathrm{C}}_{\text {meta }}$ of $\left.\mathrm{C}_{6} \mathrm{H}_{4}\right), 118.4$ (d, ${ }^{3} J=14.7, \underline{\mathrm{C}}_{\mathrm{ipso}}$ of $\left.\mathrm{C}_{6} \mathrm{H}_{4}\right), 120.6\left(\mathrm{~d},{ }^{2} J_{\mathrm{C}}=14.0, \mathrm{P}(\mathrm{F}) \mathrm{C}=\underline{\mathrm{CH}}\right), 130.8$ (s, $\underline{\mathrm{C}}_{\text {ortho }}$ of $\left.\mathrm{C}_{6} \mathrm{H}_{4}\right), 147.0$ (dd, ${ }^{1} \mathrm{~J}=258.0,{ }^{1} \mathrm{~J}=231.7$, $\mathrm{P}(\mathrm{F}) \underline{\mathrm{C}}=\mathrm{CH}), 150.4\left(\mathrm{~s}, \underline{\mathrm{C}}_{\mathrm{para}}\right.$ of $\left.\mathrm{C}_{6} \mathrm{H}_{4}\right)$.

Diethyl 1-fluoro-2-(2'-furyl)ethenephosphonate $6 \mathbf{h}: \mathrm{C}_{10} \mathrm{H}_{14} \mathrm{O}_{4} \mathrm{PF}(\mathrm{M}=248)$

E-isomer: ${ }^{31} \mathbf{P} \mathbf{N M R}+4.1\left(\mathrm{~d},{ }^{2} J_{\mathrm{PF}}=103\right)$. / $1 \mathbf{H}$ NMR $1.37\left(\mathrm{t}, 6 \mathrm{H},{ }^{3} J_{\mathrm{HH}}=7.1, \mathrm{C}_{3} \mathrm{CH}_{2} \mathrm{O}\right), 4.17(\mathrm{~m}, 4 \mathrm{H}$, $\left.\mathrm{CH}_{3} \underline{\mathrm{H}}_{2} \mathrm{O}\right), 6.48\left(\mathrm{ddd}, 1 \mathrm{H},{ }^{3} J_{\mathrm{HH}}=3.5,{ }^{3} J_{\mathrm{HH}}=1.7,{ }^{5} J_{\mathrm{HH}}=0.4, \underline{\mathrm{H}}^{4}\right.$ of $\left.\mathrm{C}_{4} \mathrm{H}_{3} \mathrm{O}\right), 6.77\left(\mathrm{dd}, 1 \mathrm{H},{ }^{3} J_{\mathrm{HFtrans}}=39.7\right.$, $\left.{ }^{3} J_{\mathrm{HPcis}}=7.8, \mathrm{P}(\mathrm{F}) \mathrm{C}=\mathrm{C} \underline{\mathrm{H}}\right), 6.79\left(\mathrm{dm}, 1 \mathrm{H},{ }^{3} J_{\mathrm{HH}}=3.5, \underline{\mathrm{H}}^{3}\right.$ of $\left.\mathrm{C}_{4} \mathrm{H}_{3} \mathrm{O}\right), 6.48\left(\mathrm{dt}, 1 \mathrm{H},{ }^{3} J_{\mathrm{HH}}=1.7,{ }^{4} J_{\mathrm{HH}}=0.8, \underline{\mathrm{H}}^{5^{\prime}}\right.$ of

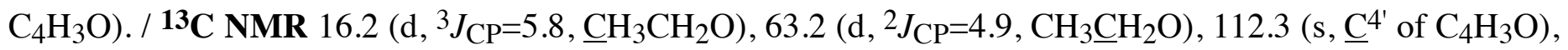
$112.9\left(\mathrm{~d},{ }^{2} J=32.3, \mathrm{P}(\mathrm{F}) \mathrm{C}=\underline{\mathrm{CH}}\right), 114.6\left(\mathrm{~d},{ }^{4} \mathrm{~J}=10.4, \underline{\mathrm{C}}^{3}\right.$ of $\left.\mathrm{C}_{4} \mathrm{H}_{3} \mathrm{O}\right), 143.9\left(\mathrm{~d},{ }^{5} \mathrm{~J}=3.9, \underline{\mathrm{C}}^{5}{ }^{1}\right.$ of $\left.\mathrm{C}_{4} \mathrm{H}_{3} \mathrm{O}\right), 146.5(\mathrm{~d}$, ${ }^{3} J=17.4, \underline{\mathrm{C}}^{2}$ of $\left.\mathrm{C}_{4} \mathrm{H}_{3} \mathrm{O}\right), 148.1\left(\mathrm{dd},{ }^{1} J=285.4,{ }^{1} J=239.3, \mathrm{P}(\mathrm{F}) \underline{\mathrm{C}}=\mathrm{CH}\right) . / \mathbf{~ m} / \mathbf{z}(\mathbf{E I}) 248\left(\mathrm{M}^{+}, 100\right), 220(9), 200$ (14), 185 (18).

Z-isomer: ${ }^{31} \mathbf{P}$ NMR +5.9 (d, $\left.{ }^{2} J_{\mathrm{PF}}=92\right) . /{ }^{1} \mathbf{H} \mathbf{N M R} 1.34\left(\mathrm{t}, 6 \mathrm{H},{ }^{3} J_{\mathrm{HH}}=7.1, \mathrm{CH}_{3} \mathrm{CH}_{2} \mathrm{O}\right), 4.16(\mathrm{~m}, 4 \mathrm{H}$, $\left.\mathrm{CH}_{3} \underline{\mathrm{C}}_{2} \mathrm{O}\right), 6.44\left(\mathrm{dd}, 1 \mathrm{H},{ }^{3} J_{\mathrm{HH}}=3.5,{ }^{3} J_{\mathrm{HH}}=1.8, \underline{\mathrm{H}}^{4}\right.$ of $\left.\mathrm{C}_{4} \mathrm{H}_{3} \mathrm{O}\right), 6.92\left(\mathrm{t}, 1 \mathrm{H},{ }^{3} J_{\mathrm{HF}}={ }^{3} J_{\mathrm{HP}}=28.3, \mathrm{P}(\mathrm{F}) \mathrm{C}=\mathrm{C} \underline{\mathrm{H}}\right)$, $7.15\left(\mathrm{dt}, 1 \mathrm{H},{ }^{3} J_{\mathrm{HH}}=3.5,{ }^{4} J_{\mathrm{HH}}=0.8, \underline{\mathrm{H}}^{3}\right.$ of $\left.\mathrm{C}_{4} \mathrm{H}_{3} \mathrm{O}\right), 7.46\left(\mathrm{~d}, 1 \mathrm{H},{ }^{3} J_{\mathrm{HH}}=1.8, \underline{\mathrm{H}}^{5}\right.$ of $\left.\mathrm{C}_{4} \mathrm{H}_{3} \mathrm{O}\right) . /{ }^{13} \mathbf{C} \mathbf{N M R} 16.6$

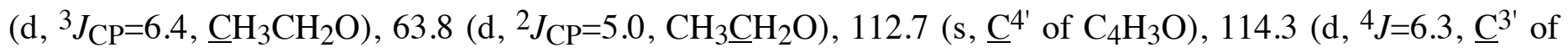
$\left.\mathrm{C}_{4} \mathrm{H}_{3} \mathrm{O}\right), 116.0\left(\mathrm{~d},{ }^{2} J=27.1, \mathrm{P}(\mathrm{F}) \mathrm{C}=\underline{\mathrm{CH}}\right), 144.1\left(\mathrm{~d},{ }^{5} \mathrm{~J}=4.4, \underline{\mathrm{C}}^{5^{\prime}}\right.$ of $\left.\mathrm{C}_{4} \mathrm{H}_{3} \mathrm{O}\right), 145.5\left(\mathrm{~d},{ }^{3} J=11.9, \underline{\mathrm{C}}^{2}\right.$ of $\left.\mathrm{C}_{4} \mathrm{H}_{3} \mathrm{O}\right)$, $149.5\left(\mathrm{dd},{ }^{1} \mathrm{~J}=267.7,{ }^{1} \mathrm{~J}=229.8, \mathrm{P}(\mathrm{F}) \underline{\mathrm{C}}=\mathrm{CH}\right)$.

Diethyl 1-fluoro-2-(2'N-methyl pyrrole)ethenephosphonate $6 \mathbf{i}: \mathrm{C}_{11} \mathrm{H}_{17} \mathrm{NO}_{3} \mathrm{PF}$

E-isomer: ${ }^{31} \mathbf{P} \mathbf{N M R}+6.9\left(\mathrm{~d},{ }^{2} \mathrm{~J}_{\mathrm{PF}}=92\right)$. / ${ }^{\mathbf{1}} \mathbf{H} \mathbf{N M R} 1.25\left(\mathrm{t}, 6 \mathrm{H},{ }^{3} \mathrm{~J}_{\mathrm{HH}}=7.1, \mathrm{CH}_{3} \mathrm{CH}_{2} \mathrm{O}\right), 3.54\left(\mathrm{~s}, 3 \mathrm{H}, \mathrm{N}-\mathrm{CH}_{3}\right)$, 3.59-4.12 (m, $\left.4 \mathrm{H}, \mathrm{CH}_{3} \underline{\mathrm{C}}_{2} \mathrm{O}\right), 6.04-6.08\left(\mathrm{~m}, 1 \mathrm{H}, \mathrm{C} \underline{\mathrm{H}}\right.$ of $\left.\mathrm{C}_{4} \mathrm{H}_{3} \mathrm{~N}\right), 6.60-6.62\left(\mathrm{~m}, 1 \mathrm{H}, \mathrm{C} \underline{\mathrm{H}}\right.$ of $\left.\mathrm{C}_{4} \mathrm{H}_{3} \mathrm{~N}\right), 6.62(\mathrm{dd}$, $\left.1 \mathrm{H},{ }^{3} J_{\mathrm{HPcis}}=8.0,{ }^{3} J_{\mathrm{HFtrans}}=39.8, \mathrm{P}(\mathrm{F}) \mathrm{C}=\mathrm{C} \underline{\mathrm{H}}\right), 6.64-6.71\left(\mathrm{~m}, 1 \mathrm{H}, \mathrm{C} \underline{\mathrm{H}}\right.$ of $\left.\mathrm{C}_{4} \mathrm{H}_{3} \mathrm{~N}\right),{ }^{13} \mathrm{C} \mathbf{~ N M R} 16.8\left(\mathrm{~d},{ }^{3} J_{\mathrm{CP}}=5.9\right.$, $\left.\underline{\mathrm{CH}}_{3} \mathrm{CH}_{2} \mathrm{O}\right), 34.8\left(\mathrm{~s}, \mathrm{~N}-\underline{\mathrm{CH}}_{3}\right), 63.6\left(\mathrm{~d},{ }^{2} J_{\mathrm{CP}}=5.1, \mathrm{CH}_{3} \underline{\mathrm{CH}}_{2} \mathrm{O}\right), 109.9\left(\mathrm{~s}, \underline{\mathrm{C}}^{4}\right.$ of $\left.\mathrm{C}_{4} \mathrm{H}_{3} \mathrm{~N}\right), 113.4\left(\mathrm{~d},{ }^{2} J=33.0\right.$, $\mathrm{P}(\mathrm{F}) \mathrm{C}=\underline{\mathrm{CH}}), 115.8\left(\mathrm{~d},{ }^{4} \mathrm{~J}=13.2, \underline{\mathrm{C}}^{3}\right.$ of $\left.\mathrm{C}_{4} \mathrm{H}_{3} \mathrm{~N}\right), 125.9\left(\mathrm{~s}, \underline{\mathrm{C}}^{5^{\prime}}\right.$ of $\left.\mathrm{C}_{4} \mathrm{H}_{3} \mathrm{~N}\right), 146.5\left(\mathrm{dd},{ }^{1} \mathrm{~J}=280.8,{ }^{1} \mathrm{~J}=241.1\right.$, $\mathrm{P}(\mathrm{F}) \underline{\mathrm{C}}=\mathrm{CH})$.

Z-isomer: ${ }^{31} \mathbf{P}$ NMR +4.6 (d, $\left.{ }^{2} J_{\mathrm{PF}}=107\right)$. / ${ }^{1} \mathbf{H}$ NMR $1.24\left(\mathrm{t}, 6 \mathrm{H},{ }^{3} J_{\mathrm{HH}}=7.1, \mathrm{C}_{3} \mathrm{CH}_{2} \mathrm{O}\right), 3.53\left(\mathrm{~s}, 3 \mathrm{H}, \mathrm{N}-\mathrm{C}_{3}\right)$, $4.08\left(\mathrm{dqd}, 4 \mathrm{H},{ }^{3} J_{\mathrm{HP}}=8.2,{ }^{3} J_{\mathrm{HH}}=7.1,{ }^{5} J_{\mathrm{HF}}=1.6, \mathrm{CH}_{3} \mathrm{CH}_{2} \mathrm{O}\right), 6.07-6.12\left(\mathrm{~m}, 1 \mathrm{H}, \mathrm{C} \underline{\mathrm{H}}\right.$ of $\left.\mathrm{C}_{4} \mathrm{H}_{3} \mathrm{~N}\right), 6.64-6.69(\mathrm{~m}$, $1 \mathrm{H}, \mathrm{C} \underline{\mathrm{H}}$ of $\left.\mathrm{C}_{4} \mathrm{H}_{3} \mathrm{~N}\right), 6.89\left(\mathrm{t}, 1 \mathrm{H},{ }^{3} J_{\mathrm{HFcis}}={ }^{3} J_{\mathrm{HPtrans}}=29.4, \mathrm{P}(\mathrm{F}) \mathrm{C}=\mathrm{C} \underline{\mathrm{H}}\right), 6.92\left(\mathrm{~d}, 1 \mathrm{H}, J=4.8, \mathrm{CH}\right.$ of $\left.\mathrm{C}_{4} \mathrm{H}_{3} \mathrm{~N}\right) /$ ${ }^{13} \mathrm{C}$ NMR $16.7\left(\mathrm{~d},{ }^{3} J_{\mathrm{CP}}=6.3, \underline{\mathrm{CH}}_{3} \mathrm{CH}_{2} \mathrm{O}\right), 34.6(\mathrm{~s}, \mathrm{~N}-\underline{\mathrm{CH}} 3), 63.6\left(\mathrm{~d},{ }^{2} J_{\mathrm{CP}}=6.0, \mathrm{CH}_{3} \underline{\mathrm{CH}}{ }_{2} \mathrm{O}\right), 109.2\left(\mathrm{~s}, \underline{\mathrm{C}}^{4}\right.$ of 
$\left.\mathrm{C}_{4} \mathrm{H}_{3} \mathrm{~N}\right), 114.3\left(\mathrm{~s}, \underline{\mathrm{C}}^{3}\right.$ of $\left.\mathrm{C}_{4} \mathrm{H}_{3} \mathrm{~N}\right), 115.8\left(\mathrm{t},{ }^{2} J=26.4, \mathrm{P}(\mathrm{F}) \mathrm{C}=\underline{\mathrm{C}} \mathrm{H}\right), 125.8\left(\mathrm{~s}, \underline{\mathrm{C}}^{5^{\prime}}\right.$ of $\left.\mathrm{C}_{4} \mathrm{H}_{3} \mathrm{~N}\right), 148.6$ (dd, $\left.{ }^{1} J=264.7,{ }^{1} J=229.7, \mathrm{P}(\mathrm{F}) \underline{\mathrm{C}}=\mathrm{CH}\right)$.

Diethyl 1-fluoro-2-(3'-thienyl)ethenephosphonate $\mathbf{6 j}$ : $\mathrm{C}_{10} \mathrm{H}_{14} \mathrm{O}_{3} \mathrm{SPF}(\mathrm{M}=264)$

E-isomer: ${ }^{31} \mathbf{P} \mathbf{N M R}+6.1\left(\mathrm{~d},{ }^{2} J_{\mathrm{PF}}=96\right)$. / ${ }^{1} \mathbf{H} \mathbf{N M R} 1.39\left(\mathrm{t}, 6 \mathrm{H},{ }^{3} \mathrm{~J}_{\mathrm{HH}}=7.1, \mathrm{CH}_{3} \mathrm{CH}_{2} \mathrm{O}\right), 4.19(\mathrm{~m}, 4 \mathrm{H}$, $\left.\mathrm{CH}_{3} \underline{\mathrm{C}}_{2} \mathrm{O}\right), 6.82\left(\mathrm{dd}, 1 \mathrm{H},{ }^{3} J_{\mathrm{HFtrans}}=41.7,{ }^{3} J_{\mathrm{HPcis}}=8.0, \mathrm{P}(\mathrm{F}) \mathrm{C}=\mathrm{C} \underline{\mathrm{H}}\right), 7.34\left(\mathrm{~s}, 2 \mathrm{H}, \underline{\mathrm{H}}^{4}\right.$ and $\underline{\mathrm{H}}^{5}$ of $\left.\mathrm{C}_{4} \mathrm{H}_{3} \mathrm{~S}\right), 7.60$ $\left(\mathrm{s}, 1 \mathrm{H}, \underline{\mathrm{H}}^{2}\right.$ of $\left.\mathrm{C}_{4} \mathrm{H}_{3} \mathrm{~S}\right) . /{ }^{13} \mathrm{C}$ NMR $16.5\left(\mathrm{~d},{ }^{3} J_{\mathrm{CP}}=6.1, \underline{\mathrm{CH}}_{3} \mathrm{CH}_{2} \mathrm{O}\right), 63.4\left(\mathrm{~d},{ }^{2} J_{\mathrm{CP}}=5.4, \mathrm{CH}_{3} \underline{\mathrm{CH}}_{2} \mathrm{O}\right), 118.0(\mathrm{~d}$, $\left.{ }^{2} J_{\mathrm{C}}=30.6, \mathrm{P}(\mathrm{F}) \mathrm{C}=\underline{\mathrm{CH}}\right), 126.4\left(\mathrm{~s}, \underline{\mathrm{C}}^{5^{\prime}}\right.$ of $\left.\mathrm{C}_{4} \mathrm{H}_{3} \mathrm{~S}\right), 128.4\left(\mathrm{~s}, \underline{\mathrm{C}}^{4^{\prime} / 2^{\prime}}\right.$ of $\left.\mathrm{C}_{4} \mathrm{H}_{3} \mathrm{~S}\right), 128.5\left(\mathrm{~s}, \underline{\mathrm{C}}^{2 / 4}\right.$ of $\left.\mathrm{C}_{4} \mathrm{H}_{3} \mathrm{~S}\right), 132.1(\mathrm{~d}$, ${ }^{3} J_{\mathrm{C}}=14.9, \underline{\mathrm{C}}^{3}$ of $\left.\mathrm{C}_{4} \mathrm{H}_{3} \mathrm{~S}\right), 148.8\left(\mathrm{dd},{ }^{1} J=283.1,{ }^{1} J=237.2, \mathrm{P}(\mathrm{F}) \underline{\mathrm{C}}=\mathrm{CH}\right)$.

Z-isomer: ${ }^{31} \mathbf{P} \mathbf{N M R}+4.2\left(\mathrm{~d},{ }^{2} J_{\mathrm{PF}}=106\right)$. / ${ }^{1} \mathbf{H}$ NMR $1.30\left(\mathrm{t}, 6 \mathrm{H},{ }^{3} \mathrm{~J}_{\mathrm{HH}}=7.1, \mathrm{C}_{3} \mathrm{CH}_{2} \mathrm{O}\right), 4.15(\mathrm{~m}, 4 \mathrm{H}$, $\left.\mathrm{CH}_{3} \underline{\mathrm{C}}_{2} \mathrm{O}\right), 7.05\left(\mathrm{t}, 1 \mathrm{H},{ }^{3} J_{\mathrm{HF}}={ }^{3} J_{\mathrm{HP}}=29.7, \mathrm{P}(\mathrm{F}) \mathrm{C}=\mathrm{C} \underline{\mathrm{H}}\right), 7.28\left(\mathrm{dd}, 1 \mathrm{H},{ }^{3} J_{\mathrm{HH}}=5.1,{ }^{4} J_{\mathrm{HH}}=2.9, \underline{\mathrm{H}}^{5}\right.$ of $\left.\mathrm{C}_{4} \mathrm{H}_{3} \mathrm{~S}\right)$, $7.43\left(\mathrm{dd}, 1 \mathrm{H},{ }^{3} J_{\mathrm{HH}}=5.1,{ }^{4} J_{\mathrm{HH}}=1.1, \underline{\mathrm{H}}^{4}\right.$ of $\left.\mathrm{C}_{4} \mathrm{H}_{3} \mathrm{~S}\right), 7.74\left(\mathrm{~d}_{\text {broad }}, 1 \mathrm{H},{ }^{4} J_{\mathrm{HH}}=2.9, \underline{\mathrm{H}}^{2}\right.$ de $\left.\mathrm{C}_{4} \mathrm{H}_{3} \mathrm{~S}\right) . /{ }^{13} \mathbf{C} \mathbf{N M R}$ $16.5\left(\mathrm{~d},{ }^{3} J_{\mathrm{CP}}=6.2, \underline{\mathrm{CH}}_{3} \mathrm{CH}_{2} \mathrm{O}\right), 63.7\left(\mathrm{~d},{ }^{2} J_{\mathrm{CP}}=5.9, \mathrm{CH}_{3} \underline{\mathrm{CH}}_{2} \mathrm{O}\right), 121.0\left(\mathrm{dd},{ }^{2} J_{\mathrm{C}}=27.7,23.2, \mathrm{P}(\mathrm{F}) \mathrm{C}=\underline{\mathrm{C}} \mathrm{H}\right), 126.0$ $\left(\mathrm{s}, \underline{\mathrm{C}}^{5^{\prime}}\right.$ of $\left.\mathrm{C}_{4} \mathrm{H}_{3} \mathrm{~S}\right), 128.0\left(\mathrm{~d},{ }^{4} J_{\mathrm{C}}=4.3, \underline{\mathrm{C}}^{4}\right.$ of $\left.\mathrm{C}_{4} \mathrm{H}_{3} \mathrm{~S}\right), 129.4\left(\mathrm{~s}, \underline{\mathrm{C}}^{2}\right.$ of $\left.\mathrm{C}_{4} \mathrm{H}_{3} \mathrm{~S}\right), 130.9\left(\mathrm{~d},{ }^{3} J_{\mathrm{C}}=9.9, \underline{\mathrm{C}}^{3}\right.$ of $\left.\mathrm{C}_{4} \mathrm{H}_{3} \mathrm{~S}\right), 150.3\left(\mathrm{dd},{ }^{1} J=265.2,{ }^{1} \mathrm{~J}=228.9, \mathrm{P}(\mathrm{F}) \underline{\mathrm{C}}=\mathrm{CH}\right) . / \mathbf{~ m} / \mathbf{z}$ (EI) $264\left(\mathrm{M}^{+}, 100\right), 235$ (17), 216 (10), 191 (33).

\section{Diethyl 1-fluoro-2-(2'-pyridyl)ethenephosphonate 6k : $\mathrm{C}_{14} \mathrm{H}_{21} \mathrm{O}_{3} \mathrm{NPF}(\mathrm{M}=301)$}

E-isomer: ${ }^{31} \mathbf{P} \mathbf{N M R}+5.6\left(\mathrm{~d},{ }^{2} J_{\mathrm{PF}}=99\right)$. / ${ }^{1} \mathbf{H}$ NMR $1.38\left(\mathrm{t}, 6 \mathrm{H},{ }^{3} J_{\mathrm{HH}}=7.1, \mathrm{C}_{3} \mathrm{CH}_{2} \mathrm{O}\right), 4.22(\mathrm{dqd}, 4 \mathrm{H}$, $\left.{ }^{3} J_{\mathrm{HP}}=8.2,{ }^{3} J_{\mathrm{HH}}=7.1,{ }^{5} J_{\mathrm{HF}}=1.6, \mathrm{CH}_{3} \underline{\mathrm{C}}_{2} \mathrm{O}\right), 6.88\left(\mathrm{dd}, 1 \mathrm{H},{ }^{3} J_{\mathrm{HFtrans}}=41.6,{ }^{3} J_{\mathrm{HPcis}}=8.7, \mathrm{P}(\mathrm{F}) \mathrm{C}=\mathrm{C} \underline{\mathrm{H}}\right), 7.23$ $\left(\mathrm{ddd}, 1 \mathrm{H},{ }^{3} J_{\mathrm{HH}}=4.8,{ }^{3} J_{\mathrm{HH}}=3.8,{ }^{4} J_{\mathrm{HH}}=1.5, \underline{\mathrm{H}}^{5}\right.$ of $\left.\mathrm{C}_{5} \mathrm{H}_{4} \mathrm{~N}\right), 7.75\left(\mathrm{~m}, 2 \mathrm{H}, \underline{\mathrm{H}}^{3}\right.$ and $\underline{\mathrm{H}}^{4}$ of $\left.\mathrm{C}_{5} \mathrm{H}_{4} \mathrm{~N}\right), 8.64(\mathrm{dm}, 1 \mathrm{H}$,

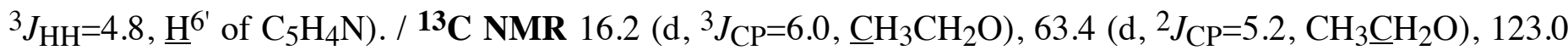
$\left(\mathrm{dd},{ }^{2} J_{\mathrm{CP}}=29.2, \mathrm{P}(\mathrm{F}) \mathrm{C}=\underline{\mathrm{C}} \mathrm{H}\right), 123.5\left(\mathrm{~s}, \underline{\mathrm{C}}^{5^{\prime}}\right.$ of $\left.\mathrm{C}_{5} \mathrm{H}_{4} \mathrm{~N}\right), 125.4\left(\mathrm{~d},{ }^{4} J_{\mathrm{CP}}=12.2, \underline{\mathrm{C}}^{3}\right.$ of $\left.\mathrm{C}_{5} \mathrm{H}_{4} \mathrm{~N}\right), 136.6\left(\mathrm{~s}, \underline{\mathrm{C}}^{4}\right.$ of $\left.\mathrm{C}_{5} \mathrm{H}_{4} \mathrm{~N}\right), 149.8\left(\mathrm{~d}, \underline{\mathrm{C}}^{6}\right.$ of $\left.\mathrm{C}_{5} \mathrm{H}_{4} \mathrm{~N}\right), 150.5\left(\mathrm{~d},{ }^{3} J=15.2, \underline{\mathrm{C}}^{2}\right.$ of $\left.\mathrm{C}_{5} \mathrm{H}_{4} \mathrm{~N}\right), 152.4\left(\mathrm{dd},{ }^{1} J=290.0,{ }^{1} J=235.1\right.$, $\mathrm{P}(\mathrm{F}) \underline{\mathrm{C}}=\mathrm{CH}) . /$ m/z (EI) $259\left(\mathrm{M}^{+}, 21\right), 230$ (31), 214 (20), 202 (70).

Z-isomer: ${ }^{31} \mathbf{P} \mathbf{N M R}+2.5\left(\mathrm{~d},{ }^{2} J_{\mathrm{PF}}=105\right) . /{ }^{1} \mathbf{H} \mathbf{N M R} 1.32\left(\mathrm{t}, 6 \mathrm{H},{ }^{3} J_{\mathrm{HH}}=7.1, \mathrm{C}_{3} \mathrm{CH}_{2} \mathrm{O}\right), 4.20(\mathrm{dqd}, 4 \mathrm{H}$, $\left.{ }^{3} J_{\mathrm{HP}}=7.8,{ }^{3} J_{\mathrm{HH}}=7.1,{ }^{5} J_{\mathrm{HF}}=2.4, \mathrm{CH}_{3} \underline{\mathrm{H}}_{2} \mathrm{O}\right), 7.19\left(\mathrm{t}, 1 \mathrm{H},{ }^{3} J=28.2, \mathrm{P}(\mathrm{F}) \mathrm{C}=\mathrm{C} \underline{\mathrm{H}}\right), 7.27\left(\mathrm{ddd}, 1 \mathrm{H},{ }^{3} J_{\mathrm{HH}}=4.8\right.$, ${ }^{3} J_{\mathrm{HH}}=3.8,{ }^{4} J_{\mathrm{HH}}=2.1, \underline{\mathrm{H}}^{5}$ of $\left.\mathrm{C}_{5} \mathrm{H}_{4} \mathrm{~N}\right), 7.74\left(\mathrm{~m}, 2 \mathrm{H}, \underline{\mathrm{H}}^{3}\right.$ and $\underline{\mathrm{H}}^{4}$ of $\left.\mathrm{C}_{5} \mathrm{H}_{4} \mathrm{~N}\right), 8.66\left(\mathrm{dm}, 1 \mathrm{H},{ }^{3} J_{\mathrm{HH}}=4.8, \underline{\mathrm{H}}^{6}\right.$ of $\left.\mathrm{C}_{5} \mathrm{H}_{4} \mathrm{~N}\right) . /{ }^{13} \mathrm{C}$ NMR $16.7\left(\mathrm{~d},{ }^{3} J_{\mathrm{CP}}=6.3, \underline{\mathrm{CH}}_{3} \mathrm{CH}_{2} \mathrm{O}\right), 64.1\left(\mathrm{~d},{ }^{2} J_{\mathrm{CP}}=6.0, \mathrm{CH}_{3} \underline{C H}_{2} \mathrm{O}\right), 123.6\left(\mathrm{~s}, \underline{\mathrm{C}}^{5}\right.$ of $\left.\mathrm{C}_{5} \mathrm{H}_{4} \mathrm{~N}\right)$, $125.6\left(\mathrm{~s}\right.$ broad,$\underline{\mathrm{C}}^{3}$ of $\left.\mathrm{C}_{5} \mathrm{H}_{4} \mathrm{~N}\right), 126.2\left(\mathrm{dd},{ }^{2} J_{\mathrm{C}}=26.2,20.3, \mathrm{P}(\mathrm{F}) \mathrm{C}=\underline{\mathrm{CH}}\right), 136.7\left(\mathrm{~s}, \underline{\mathrm{C}}^{4}\right.$ of $\left.\mathrm{C}_{5} \mathrm{H}_{4} \mathrm{~N}\right), 149.9\left(\mathrm{~s}, \underline{\mathrm{C}}^{6}\right.$ of $\left.\mathrm{C}_{5} \mathrm{H}_{4} \mathrm{~N}\right), 151.0\left(\mathrm{~d},{ }^{3} J_{\mathrm{CP}}=13.4, \underline{\mathrm{C}}^{2}\right.$ of $\left.\mathrm{C}_{5} \mathrm{H}_{4} \mathrm{~N}\right), 154.2\left(\mathrm{dd},{ }^{1} J=274.5,{ }^{1} J=227.2, \mathrm{P}(\mathrm{F}) \underline{\mathrm{C}}=\mathrm{CH}\right)$.

Diethyl 1-fluoro-2-methyl-2-(4'-bromo)phenylethenephosphonate $6 \mathbf{1}$ : $\mathrm{C}_{13} \mathrm{H}_{17} \mathrm{O}_{3} \mathrm{PFBr}(\mathrm{M}=351)$

E-isomer: ${ }^{31} \mathbf{P} \mathbf{N M R}+5.1\left(\mathrm{~d},{ }^{2} J_{\mathrm{PF}}=97\right)$. / ${ }^{1} \mathbf{H}$ NMR $1.19\left(\mathrm{t}, 6 \mathrm{H},{ }^{3} \mathrm{~J}_{\mathrm{HH}}=7.0, \mathrm{C}_{3} \mathrm{CH}_{2} \mathrm{O}\right), 2.11\left(\mathrm{~m}, 3 \mathrm{H}, \mathrm{CH}_{3}\right)$, $3.94\left(\mathrm{~m}, 4 \mathrm{H}, \mathrm{CH}_{3} \underline{\mathrm{C}}_{2} \mathrm{O}\right), 7.15\left(\mathrm{~d}, 2 \mathrm{H},{ }^{3} J_{\mathrm{HH}}=7.6, \underline{\mathrm{H}}_{\text {ortho }}\right.$ of $\left.\mathrm{C}_{6} \mathrm{H}_{4}\right), 7.46\left(\mathrm{~d}, 2 \mathrm{H},{ }^{3} \mathrm{~J}_{\mathrm{HH}}=7.6, \underline{\mathrm{H}}_{\mathrm{meta}}\right.$ of $\left.\mathrm{C}_{6} \mathrm{H}_{4}\right)$. /

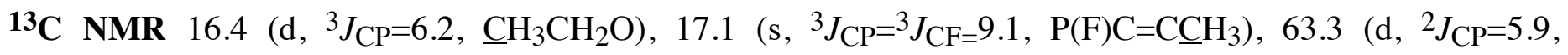
$\mathrm{CH}_{3} \underline{\mathrm{CH}}_{2} \mathrm{O}$ ), 122.7 (s, $\underline{\mathrm{C}}_{\text {para }}$ of $\mathrm{C}_{6} \mathrm{H}_{4} \mathrm{Br}$ ), 129.8 (d, ${ }^{4} \mathrm{~J}=4.5, \underline{\mathrm{C}}_{\text {ortho }}$ of $\mathrm{C}_{6} \mathrm{H}_{4} \mathrm{Br}$ ), 131.6 (s, $\underline{\mathrm{C}}_{\text {meta }}$ of $\mathrm{C}_{6} \mathrm{H}_{4} \mathrm{Br}$ ), $132.8\left(\mathrm{dd},{ }^{2} J=31.6,{ }^{2} J=5.9, \mathrm{P}(\mathrm{F}) \mathrm{C}=\underline{\mathrm{C}}\right), 135.7\left(\mathrm{dd},{ }^{3} J=13.7,{ }^{3} J=2.9, \underline{\mathrm{C}}_{\text {ipso }}\right.$ of $\left.\mathrm{C}_{6} \mathrm{H}_{4} \mathrm{Br}\right), 146.0\left(\mathrm{dd},{ }^{1} J=268.5\right.$, $\left.{ }^{1} J=233.6, \mathrm{P}(\mathrm{F}) \underline{\mathrm{C}}=\mathrm{C}\right)$.

Z-isomer: 31P NMR +3.8 (d, $\left.{ }^{2} J_{\mathrm{PF}}=111\right)$. / ${ }^{1} \mathbf{H}$ NMR $1.39\left(\mathrm{t}, 6 \mathrm{H},{ }^{3} J_{\mathrm{HH}}=7.0, \mathrm{C}_{3} \mathrm{CH}_{2} \mathrm{O}\right), 2.35\left(\mathrm{~m}, 3 \mathrm{H}, \mathrm{C}_{3}\right)$, $4.21\left(\mathrm{p}, 4 \mathrm{H},{ }^{3} J_{\mathrm{HH}}=7.1,{ }^{3} J_{\mathrm{HP}}=7.1, \mathrm{CH}_{3} \mathrm{CH}_{2} \mathrm{O}\right), 7.17\left(\mathrm{~d}, 2 \mathrm{H},{ }^{3} J_{\mathrm{HH}}=7.2, \underline{\mathrm{H}}_{\mathrm{ortho}}\right.$ of $\left.\mathrm{C}_{6} \mathrm{H}_{4}\right), 7.48\left(\mathrm{~d}, 2 \mathrm{H},{ }^{3} J_{\mathrm{HH}}=7.2\right.$,

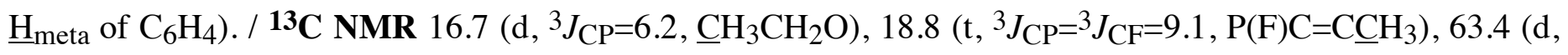
${ }^{2} J_{\mathrm{CP}}=6.0, \mathrm{CH}_{3} \underline{\mathrm{C}} \mathrm{H}_{2} \mathrm{O}$ ), 123.0 (s, $\underline{\mathrm{C}}_{\text {para }}$ of $\mathrm{C}_{6} \mathrm{H}_{4} \mathrm{Br}$ ), 130.6 (s, $\underline{\mathrm{C}}_{\text {ortho }}$ of $\mathrm{C}_{6} \mathrm{H}_{4} \mathrm{Br}$ ), 131.9 (s, $\underline{\mathrm{C}}_{\text {meta }}$ of $\mathrm{C}_{6} \mathrm{H}_{4} \mathrm{Br}$ ), 
$134.1\left(\mathrm{dd},{ }^{2} J=27.3,{ }^{2} J=13.4, \mathrm{P}(\mathrm{F}) \mathrm{C}=\underline{\mathrm{C}}\right), 136.6\left(\mathrm{~d},{ }^{2} J=7.9, \underline{\mathrm{C}}_{\text {ipso }}\right.$ of $\left.\mathrm{C}_{6} \mathrm{H}_{4} \mathrm{Br}\right), 148.1\left(\mathrm{dd},{ }^{1} J=267.2,{ }^{1} J=239.4\right.$, $\mathrm{P}(\mathrm{F}) \underline{\mathrm{C}}=\mathrm{C})$.

Diethyl 1-fluoro-2-cyclopropyl-2-methylethenephosphonate $6 \mathbf{m}$ : $\mathrm{C}_{10} \mathrm{H}_{18} \mathrm{O}_{3} \mathrm{PF}(\mathrm{M}=236)$

E-isomer: ${ }^{31} \mathbf{P}$ NMR +6.3 (d, $\left.{ }^{2} J_{\mathrm{PF}}=104\right)$. / ${ }^{1} \mathbf{H}$ NMR 0.6-0.8 (m, 4H, A, A', B and B' parts of AA'BB'X system, $\left.\mathrm{CH}_{X}\left(\mathrm{C}_{2}\right)_{2}\right), 1.34\left(\mathrm{t}, 6 \mathrm{H},{ }^{3} J_{\mathrm{HH}}=7.0, \mathrm{C}_{3} \mathrm{CH}_{2} \mathrm{O}\right), 1.42\left(\mathrm{~m}, 3 \mathrm{H}, \mathrm{C}_{3}\right), 2.66(\mathrm{~m}, 1 \mathrm{H}, \mathrm{X}$ part of AA'BB'X system, $\left.\underline{\mathrm{H}}_{\mathrm{X}}\left(\mathrm{CH}_{2}\right)_{2}\right), 4.15\left(\mathrm{~m}, 4 \mathrm{H}, \mathrm{CH}_{3} \underline{\mathrm{C}}_{2} \mathrm{O}\right) . /{ }^{13} \mathbf{C} \mathbf{N M R} 4.6\left(\mathrm{~s}, \mathrm{CH}\left(\underline{\mathrm{CH}}_{2}\right)_{2}\right), 10.4\left(\mathrm{~d},{ }^{3} J=4.0\right.$,

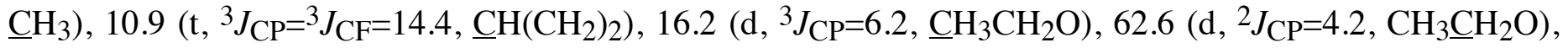
$138.0\left(\mathrm{dd},{ }^{2} J=30.5,{ }^{2} J=8.9, \mathrm{P}(\mathrm{F}) \mathrm{C}=\underline{\mathrm{C}}\right), 144.8\left(\mathrm{dd},{ }^{1} J=256.2,{ }^{1} J=236.3, \mathrm{P}(\mathrm{F}) \underline{\mathrm{C}}=\mathrm{C}\right) . / \mathbf{m} / \mathbf{z}(\mathbf{E I}) 236\left(\mathrm{M}^{+}, 43\right)$, $208(46), 180(60)$.

Z-isomer: 31P NMR + $5.8\left(\mathrm{~d},{ }^{2} J_{\mathrm{PF}}=104\right) .{ }^{1} \mathbf{H}$ NMR 0.6-0.8 $\left(\mathrm{m}, 4 \mathrm{H}, \mathrm{A}, \mathrm{A}^{\prime}, \mathrm{B}\right.$ and $\mathrm{B}^{\prime}$ parts of AA'BB'X system, $\left.\mathrm{CH}_{X}\left(\mathrm{C}_{2}\right)_{2}\right), 1.34\left(\mathrm{t}, 6 \mathrm{H},{ }^{3} J_{\mathrm{HH}}=7.0, \mathrm{C}_{3} \mathrm{CH}_{2} \mathrm{O}\right), 1.67\left(\mathrm{~m}, 3 \mathrm{H}, \mathrm{C}_{3}\right), 2.09(\mathrm{~m}, 1 \mathrm{H}, \mathrm{X}$ part of AA'BB'X system, $\left.\underline{\mathrm{C}}_{\mathrm{X}}\left(\mathrm{CH}_{2}\right)_{2}\right), 4.15\left(\mathrm{~m}, 4 \mathrm{H}, \mathrm{CH}_{3} \underline{\mathrm{C}}_{2} \mathrm{O}\right) . /{ }^{13} \mathbf{C} \mathbf{N M R} 5.0 \quad\left(\mathrm{~s}, \mathrm{CH}\left(\underline{\mathrm{CH}}_{2}\right)_{2}\right), 8.6$ (t,

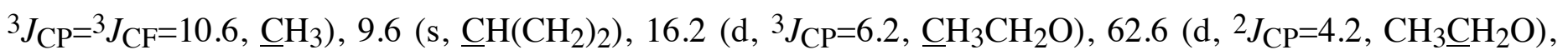
$137.2\left(\mathrm{dd},{ }^{2} J=30.6,{ }^{2} J=5.9, \mathrm{P}(\mathrm{F}) \mathrm{C}=\underline{\mathrm{C}}\right), 145.6\left(\mathrm{dd},{ }^{1} J=256.5,{ }^{1} J=238.2, \mathrm{P}(\mathrm{F}) \underline{\mathrm{C}}=\mathrm{C}\right) . / \mathrm{m} / \mathbf{z}(\mathbf{E I}) 236\left(\mathrm{M}^{+}, 62\right)$, 208 (43), 180 (52).

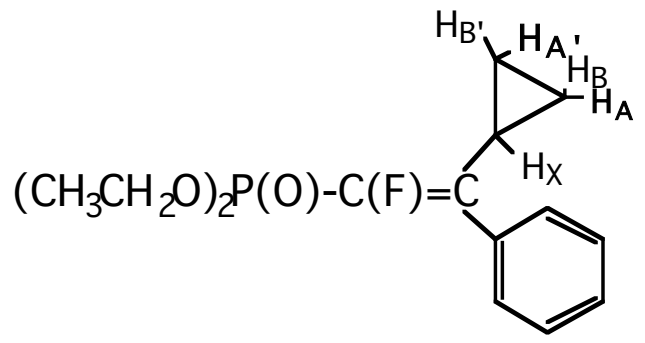

Diethyl 1-fluoro-2-cyclopropyl-2-phenylethenephosphonate 6n : $\mathrm{C}_{15} \mathrm{H}_{20} \mathrm{O}_{3} \mathrm{PF}(\mathrm{M}=298)$

E-isomer: ${ }^{31} \mathbf{P}$ NMR +6.6 (d, $\left.{ }^{2} J_{\mathrm{PF}}=106\right)$. / ${ }^{1} \mathbf{H}$ NMR $0.30\left(\mathrm{~m}, 2 \mathrm{H}, \mathrm{B}\right.$ and $\mathrm{B}^{\prime}$ parts of AA'BB'X system, $\left.\mathrm{CH}_{\mathrm{X}}\left(\mathrm{CH}_{\mathrm{A}} \mathrm{H}_{\mathrm{B}}\right)_{2}\right), 0.75\left(\mathrm{~m}, 2 \mathrm{H}, \mathrm{A}\right.$ and $\mathrm{A}^{\prime}$ parts of $\mathrm{AA}^{\prime} \mathrm{BB}^{\prime} \mathrm{X}$ system, $\left.\mathrm{CH}_{\mathrm{X}}\left(\mathrm{CH}_{\mathrm{A}} \mathrm{H}_{\mathrm{B}}\right)_{2}\right), 1.39\left(\mathrm{t}, 6 \mathrm{H},{ }^{3} J_{\mathrm{HH}}=7.1\right.$, $\left.\mathrm{C}_{3} \mathrm{CH}_{2} \mathrm{O}\right), 2.77\left(\mathrm{~m}, 1 \mathrm{H}, \mathrm{X}\right.$ part of $\mathrm{AA}^{\prime} \mathrm{BB}^{\prime} \mathrm{X}$ system, $\left.\mathrm{C}_{\mathrm{X}}\left(\mathrm{CH}_{\mathrm{A}} \mathrm{H}_{\mathrm{B}}\right)_{2}\right), 4.23\left(\mathrm{qd}, 4 \mathrm{H},{ }^{3} J_{\mathrm{HH}}=7.1,{ }^{3} J_{\mathrm{HP}}=7.1\right.$, $\left.\mathrm{CH}_{3} \underline{\mathrm{C}}_{2} \mathrm{O}\right), 7.03\left(\mathrm{~m}, 2 \mathrm{H}, \underline{\mathrm{H}}_{\text {meta }}\right.$ of $\left.\mathrm{C}_{6} \mathrm{H}_{5}\right), 7.32\left(\mathrm{~m}, 3 \mathrm{H}, \underline{\mathrm{H}}_{\text {ortho }}\right.$ and $\underline{\mathrm{H}}_{\text {para }}$ of $\left.\mathrm{C}_{6} \mathrm{H}_{5}\right)$. / 13C NMR 5.1 (s, $\left.\mathrm{CH}\left(\underline{\mathrm{CH}_{2}}\right)_{2}\right), 10.9$ (s, $\left.\underline{\mathrm{CH}}\left(\mathrm{CH}_{2}\right)_{2}\right), 16.7\left(\mathrm{~d},{ }^{3} J_{\mathrm{CP}}=6.6, \underline{\mathrm{CH}}_{3} \mathrm{CH}_{2} \mathrm{O}\right), 63.4\left(\mathrm{~d},{ }^{2} J_{\mathrm{CP}}=5.8, \mathrm{CH}_{3} \underline{\mathrm{CH}}_{2} \mathrm{O}\right), 128.3$ (s, $\underline{\mathrm{C}}_{\text {para }}$ of $\mathrm{C}_{6} \mathrm{H}_{5}$ ), 128.4 (s, $\underline{\mathrm{C}}_{\text {meta }}$ of $\mathrm{C}_{6} \mathrm{H}_{5}$ ), 139.1 (s sroad,$\underline{\mathrm{C}}_{\text {ortho }}$ of $\mathrm{C}_{6} \mathrm{H}_{5}$ ), 132.1 (dd, ${ }^{3} J=12.2,{ }^{3} J=4.7, \underline{\mathrm{C}}_{\mathrm{ipso}}$ of $\left.\mathrm{C}_{6} \mathrm{H}_{5}\right), 142.6\left(\mathrm{dd},{ }^{2} J=31.0,{ }^{2} J=9.6, \mathrm{P}(\mathrm{F}) \mathrm{C}=\underline{\mathrm{C}}\right), 145.4\left(\mathrm{dd},{ }^{1} J=260.0,{ }^{1} J=231.4, \mathrm{P}(\mathrm{F}) \underline{\mathrm{C}}=\mathrm{C}\right)$.

Z-isomer: ${ }^{31} \mathbf{P}$ NMR +5.1 (d, $\left.{ }^{2} J_{\mathrm{PF}}=112\right)$. / ${ }^{1} \mathbf{H}$ NMR $0.32\left(\mathrm{~m}, 2 \mathrm{H}, \mathrm{B}\right.$ and $\mathrm{B}^{\prime}$ parts of AA'BB'X system, $\left.\mathrm{CH}_{\mathrm{X}}\left(\mathrm{CH}_{\mathrm{A}} \underline{\mathrm{H}}_{\mathrm{B}}\right)_{2}\right), 0.74\left(\mathrm{~m}, 2 \mathrm{H}, \mathrm{A}\right.$ and $\mathrm{A}^{\prime}$ parts of $\mathrm{AA}^{\prime} \mathrm{BB}$ 'X system, $\left.\mathrm{CH}_{\mathrm{X}}\left(\mathrm{C}_{\mathrm{A}} \mathrm{H}_{\mathrm{B}}\right)_{2}\right), 1.16\left(\mathrm{t}, 6 \mathrm{H},{ }^{3} J_{\mathrm{HH}}=7.1\right.$, $\left.\mathrm{CH}_{3} \mathrm{CH}_{2} \mathrm{O}\right), 2.22\left(\mathrm{~m}, 1 \mathrm{H}, \mathrm{X}\right.$ part of $\mathrm{AA}^{\prime} \mathrm{BB}^{\prime} \mathrm{X}$ system, $\left.\mathrm{CHX}\left(\mathrm{CH}_{\mathrm{A}} \mathrm{H}_{\mathrm{B}}\right)_{2}\right), 3.88\left(\mathrm{~m}, 4 \mathrm{H}, \mathrm{CH}_{3} \mathrm{CH}_{2} \mathrm{O}\right), 7.09$ (m, $2 \mathrm{H}, \underline{\mathrm{H}}_{\text {meta }}$ of $\left.\mathrm{C}_{6} \mathrm{H}_{5}\right), 7.30\left(\mathrm{~m}, 2 \mathrm{H}, \mathrm{H}_{\text {ortho }}\right.$ and $\mathrm{H}_{\text {para }}$ of $\left.\mathrm{C}_{6} \mathrm{H}_{5}\right) . /{ }^{13} \mathbf{C} \mathbf{N M R} 5.3\left(\mathrm{~s}, \mathrm{CH}\left(\underline{\mathrm{CH}}_{2}\right)_{2}\right), 11.7\left(\mathrm{t},{ }^{3} \mathrm{~J}=11.9\right.$,

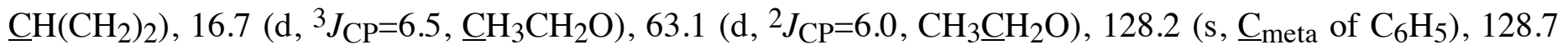
(s, $\underline{\mathrm{C}}_{\text {para }}$ of $\left.\mathrm{C}_{6} \mathrm{H}_{5}\right), 130.6$ (s $\left.{ }^{2} J=8.6, \mathrm{P}(\mathrm{F}) \mathrm{C}=\underline{\mathrm{C}}\right), 148.4\left(\mathrm{dd},{ }^{1} J=265.5,{ }^{1} J=242.9, \mathrm{P}(\mathrm{F}) \underline{\mathrm{C}}=\mathrm{C}\right)$.

Diethyl 1-fluoro-2-(1'-methyl)ethyl-2-methylethenephosphonate $6 \mathbf{0}: \mathrm{C}_{10} \mathrm{H}_{20} \mathrm{O}_{3} \mathrm{PF}(\mathrm{M}=238)$

E-isomer: ${ }^{31} \mathbf{P} \mathbf{N M R}+6.2\left(\mathrm{~d},{ }^{2} J_{\mathrm{PF}}=110\right)$. / $\mathbf{1}_{\mathbf{H}} \mathbf{N M R} 0.98\left(\mathrm{~d}, 6 \mathrm{H},{ }^{3} J_{\mathrm{HH}}=6.8, \mathrm{CH}\left(\mathrm{C}_{3}\right)_{2}\right), 1.31(\mathrm{t}, 6 \mathrm{H}$, $\left.{ }^{3} J_{\mathrm{HH}}=7.0, \mathrm{CH}_{3} \mathrm{CH}{ }_{2} \mathrm{O}\right), 1.90\left(\mathrm{t}, 3 \mathrm{H},{ }^{4} J_{\mathrm{HP}}={ }^{4} J_{\mathrm{HF}}=3.2,=\mathrm{C}-\mathrm{CH}_{3}\right), 3.13\left(\mathrm{pm}, 1 \mathrm{H},{ }^{3} J_{\mathrm{HH}}=6.8, \mathrm{CH}\left(\mathrm{CH}_{3}\right)_{2}\right), 4.09(\mathrm{~m}$,

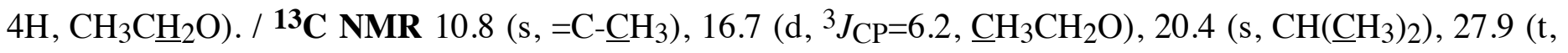


$\left.{ }^{3} J_{\mathrm{CP}}={ }^{3} J_{\mathrm{CF}}=9.8, \underline{\mathrm{C}} \mathrm{H}\left(\mathrm{CH}_{3}\right)_{2}\right), 63.1\left(\mathrm{~d},{ }^{2} J_{\mathrm{CP}}=5.8, \mathrm{CH}_{3} \underline{\mathrm{CH}}_{2} \mathrm{O}\right), 142.1\left(\mathrm{dd},{ }^{2} J=27.5,{ }^{2} J=7.0, \mathrm{P}(\mathrm{F}) \mathrm{C}=\underline{\mathrm{C}}\right), 144.0(\mathrm{dd}$, $\left.{ }^{1} J=257.1,{ }^{1} J=235.8, \mathrm{P}(\mathrm{F}) \underline{\mathrm{C}}=\mathrm{C}\right)$.

Z-isomer: ${ }^{31} \mathbf{P} \mathbf{N M R}+5.8\left(\mathrm{~d},{ }^{2} J_{\mathrm{PF}}=109\right)$. / $\mathbf{1}_{\mathbf{H}} \mathbf{N M R} 1.02\left(\mathrm{~d}, 6 \mathrm{H},{ }^{3} J_{\mathrm{HH}}=6.8, \mathrm{CH}\left(\mathrm{C}_{3}\right)_{2}\right), 1.34(\mathrm{t}, 6 \mathrm{H}$, $\left.{ }^{3} J_{\mathrm{HH}}=7.1, \mathrm{C}_{3} \mathrm{CH}_{2} \mathrm{O}\right), 1.72\left(\mathrm{dd}, 3 \mathrm{H},{ }^{4} \mathrm{~J}=4.4,{ }^{4} \mathrm{~J}=2.4,=\mathrm{C}-\underline{\mathrm{H}}_{3}\right), 3.57\left(\mathrm{pd}, 1 \mathrm{H},{ }^{3} J_{\mathrm{HH}}=6.8,{ }^{3} J_{\mathrm{HH}}=3.1\right.$, $\left.\mathrm{C} \underline{\mathrm{H}}\left(\mathrm{CH}_{3}\right)_{2}\right), 4.12\left(\mathrm{~m}, 4 \mathrm{H}, \mathrm{CH}_{3} \underline{\mathrm{C}}_{2} \mathrm{O}\right) . /{ }^{13} \mathrm{C}$ NMR $9.3\left(\mathrm{t},{ }^{3} J_{\mathrm{CP}}={ }^{3} J_{\mathrm{CF}}=10.9,=\mathrm{C}-\underline{\mathrm{CH}_{3}}\right), 16.6\left(\mathrm{~d},{ }^{3} J_{\mathrm{CP}}=6.4\right.$, $\left.\underline{\mathrm{CH}}_{3} \mathrm{CH}_{2} \mathrm{O}\right), 21.0\left(\mathrm{~s}, \mathrm{CH}\left(\underline{\mathrm{CH}}_{3}\right)_{2}\right), 27.6\left(\mathrm{~s}, \underline{\mathrm{CH}}\left(\mathrm{CH}_{3}\right)_{2}\right), 62.9$ (d, $\left.{ }^{2} J_{\mathrm{CP}}=5.1, \mathrm{CH}_{3} \underline{\mathrm{CH}}_{2} \mathrm{O}\right), 142.1$ (dd, ${ }^{2} J=31.8$, $\left.{ }^{2} J=4.6, \mathrm{P}(\mathrm{F}) \mathrm{C}=\underline{\mathrm{C}}\right), 144.6\left(\mathrm{dd},{ }^{1} J=259.5,{ }^{1} \mathrm{~J}=236.5, \mathrm{P}(\mathrm{F}) \underline{\mathrm{C}}=\mathrm{C}\right)$.

Diethyl 1-[5'-(R)-(-)-isopropenyl-2'-methyl]cyclohex-2'-enylidene-1-fluoromethane-phosphonate $\mathbf{6 p}(\boldsymbol{Z}) \quad$ : $\mathrm{C}_{15} \mathrm{H}_{26} \mathrm{O}_{3} \mathrm{PF}(\mathrm{M}=304)$

31P NMR +6.5 (d, $\left.{ }^{2} J_{\mathrm{PF}}=116\right) . /{ }^{1} \mathbf{H}$ NMR $1.34\left(\mathrm{t}, 6 \mathrm{H},{ }^{3} \mathrm{~J}_{\mathrm{HH}}=7.0, \mathrm{CH}_{3} \mathrm{CH}_{2} \mathrm{O}\right), 1.73\left(\mathrm{~s}, 3 \mathrm{H}, \mathrm{C}\left(\mathrm{CH}_{2}\right) \mathrm{CH}_{3}\right), 2.05$ $\left(\mathrm{m}, 2 \mathrm{H},{ }^{4} \mathrm{C}_{2}\right), 2.13\left(\mathrm{~s}, 3 \mathrm{H},{ }^{2} \mathrm{C}-\mathrm{CH}_{3}\right), 2.30\left(\mathrm{dd}, 2 \mathrm{H},{ }^{3} \mathrm{~J}_{\mathrm{HH}}=11.4,{ }^{4} \mathrm{~J}=3.2, \mathrm{P}(\mathrm{F}) \mathrm{C}=\mathrm{C}-{ }^{6} \mathrm{CH}_{2}\right), 2.93(\mathrm{dm}, 1 \mathrm{H}$, $\left.{ }^{3} J_{\mathrm{HH}}=11.4,{ }^{5} \mathrm{C} \underline{\mathrm{H}}\right), 4.15\left(\mathrm{~m}, 4 \mathrm{H}, \mathrm{CH}_{3} \underline{\mathrm{H}}_{2} \mathrm{O}\right), 4.74\left(\mathrm{~d}, 2 \mathrm{H},{ }^{4} J_{\mathrm{HH}}=3.6, \mathrm{C}\left(\mathrm{C}_{2}\right) \mathrm{CH}_{3}\right), 5.81$ (sbroad, $\left.1 \mathrm{H},={ }^{3} \mathrm{C} \underline{\mathrm{H}}\right)$. /

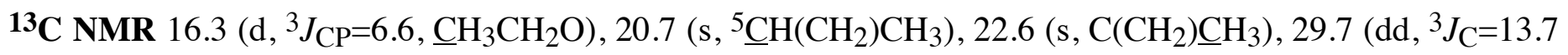
et $\left.9.0, \mathrm{P}(\mathrm{F}) \mathrm{C}=\mathrm{C}-{ }^{6} \underline{\mathrm{C}} \mathrm{H}_{2}\right), 31.8\left(\mathrm{~s},{ }^{4} \underline{\mathrm{CH}}_{2}\right), 40.5\left(\mathrm{~d},{ }^{4} \mathrm{~J}_{\mathrm{C}}=2.0,{ }^{2} \mathrm{C}-\underline{\mathrm{CH}}_{3}\right), 63.1\left(\mathrm{~d},{ }^{2} J_{\mathrm{CP}}=6.0, \mathrm{CH}_{3} \underline{\mathrm{C}} \mathrm{H}_{2} \mathrm{O}\right), 110.0(\mathrm{~s}$, $\left.{ }^{5} \mathrm{C}-\mathrm{C}\left(\mathrm{CH}_{3}\right)=\mathrm{CH}_{2}\right), 128.6\left(\mathrm{t},{ }^{3} J_{\mathrm{C}}=3.5,{ }^{2} \underline{\mathrm{C}}\right), 133.6\left(\mathrm{~d},{ }^{4} J_{\mathrm{C}}=8.8,{ }^{3} \underline{\mathrm{CH}}\right) .136 .2\left(\mathrm{dd},{ }^{2} J=25.7,{ }^{2} J=12.6, \mathrm{P}(\mathrm{F}) \mathrm{C}={ }^{1} \underline{\mathrm{C}}\right)$, $146.2\left(\mathrm{dd},{ }^{1} \mathrm{~J}=258.6,{ }^{1} \mathrm{~J}=235.6, \mathrm{P}(\mathrm{F}) \underline{\mathrm{C}}\right), 148.0\left(\mathrm{~s},{ }^{5} \mathrm{CH}-\underline{\mathrm{C}}\left(\mathrm{CH}_{2}\right) \mathrm{CH}_{3}\right)$.

Diethyl 1-[3',5',5'-trimethyl]cyclohex-2'-enylidene-1-fluoro-methanephosphonate $\quad \mathbf{6 q} \quad(\boldsymbol{Z}): \quad \mathrm{C}_{14} \mathrm{H}_{24} \mathrm{O}_{3} \mathrm{PF}$ (M=290)

31P NMR +4.1 (d, $\left.{ }^{2} J_{\mathrm{PF}}=104\right) . /{ }^{1} \mathbf{H}$ NMR $0.96\left(\mathrm{~s}, 6 \mathrm{H}, \mathrm{C}\left(\mathrm{C}_{3}\right)_{2}\right), 1.35\left(\mathrm{t}, 6 \mathrm{H},{ }^{3} J_{\mathrm{HH}}=7.1, \mathrm{C}_{3} \mathrm{CH}_{2} \mathrm{O}\right), 1.82(\mathrm{~s}$, $\left.3 \mathrm{H},=\mathrm{CC} \underline{H}_{3}\right), 1.92\left(\mathrm{~s}, 2 \mathrm{H}, \mathrm{CH}^{4}{ }_{2}\right), 2.25\left(\mathrm{t}, 2 \mathrm{H},{ }^{4} J_{\mathrm{HF}}={ }^{4} J_{\mathrm{HP}}=2.5, \mathrm{C}^{6}{ }^{\prime}{ }^{2}\right), 4.14\left(\mathrm{~m}, 4 \mathrm{H}, \mathrm{CH}_{3} \mathrm{C} \underline{H}_{2} \mathrm{O}\right), 6.83$ (s $\left.1 \mathrm{H}, \mathrm{C}^{2}{ }^{\prime}\right) . /{ }^{13} \mathrm{C}$ NMR $16.5\left(\mathrm{~d},{ }^{3} J_{\mathrm{CP}}=6.7, \underline{\mathrm{C}}{ }_{3} \mathrm{CH}_{2} \mathrm{O}\right), 25.0\left(\mathrm{~s},=\mathrm{C}_{\mathrm{CH}}\right), 28.5\left(\mathrm{~s}, \mathrm{C}\left(\underline{\mathrm{CH}}_{3}\right)_{2}\right), 30.5(\mathrm{~s}$, $\left.\mathrm{C}^{5}\left(\underline{\mathrm{CH}}_{3}\right)_{2}\right), 36.0\left(\mathrm{t},{ }^{3} J_{\mathrm{CF}}={ }^{3} J_{\mathrm{CP}}=8.8, \underline{\mathrm{C}}^{6} \mathrm{H}_{2}\right), 45.0\left(\mathrm{~s}, \underline{\mathrm{C}}^{4} \mathrm{H}_{2}\right), 63.2\left(\mathrm{~d},{ }^{2} J_{\mathrm{CP}}=4.7, \mathrm{CH}_{3} \underline{\mathrm{CH}}{ }_{2} \mathrm{O}\right), 116.0$ (s, $\left.=\mathrm{C}^{3} \mathrm{CH}_{3}\right), 135.6\left(\mathrm{dd},{ }^{2} J=28.9,10.8, \mathrm{P}(\mathrm{F}) \mathrm{C}=\underline{\mathrm{C}}^{1}\right), 143.7\left(\mathrm{dd},{ }^{3} J=12.1{ }^{2} J=10.0, \underline{\mathrm{C}}^{2} \mathrm{H}\right), 144.2\left(\mathrm{dd},{ }^{1} J=259.3\right.$, $\left.{ }^{1} J=236.4, \mathrm{P}(\mathrm{F}) \underline{\mathrm{C}}\right) . / \mathbf{~ m} / \mathbf{z}(\mathbf{E I}) 290\left(\mathrm{M}^{+}, 33\right), 275$ (100), 247 (37), 219 (52).

Diethyl 1-fluoro 1-(1',2',3',4'-tetrahydro-2'-naphthylidenemethylphosphonate $\mathbf{6 r}(\boldsymbol{Z}): \mathrm{C}_{15} \mathrm{H}_{20} \mathrm{O}_{3} \mathrm{PF}(\mathrm{M}=298)$ 31P NMR +6.3 (d, $\left.{ }^{2} J_{\mathrm{PF}}=116\right) . /{ }^{1} \mathbf{H}$ NMR $1.15\left(\mathrm{t}, 6 \mathrm{H},{ }^{3} J_{\mathrm{HH}}=7.1, \mathrm{CH}_{3} \mathrm{CH}_{2} \mathrm{O}\right), 1.35\left(\mathrm{dt}, 2 \mathrm{H}, J=9.4,{ }^{3} J_{\mathrm{HH}}=7.1\right.$, $\left.\mathrm{C}^{3} \mathrm{C}_{\mathrm{H}_{2}}\right), 1.78$ (p, 2H, J=6.6, $\left.\mathrm{C}^{2} \underline{\mathrm{H}}_{2}\right), 2.65$ (m, 2H, $\left.\mathrm{C}^{4} \underline{\mathrm{H}}_{2}\right), 4.00$ (m, 4H, $\mathrm{CH}_{3} \mathrm{C}_{2} \mathrm{O}$ ), 7.10-7.30 (m, 3H, $\mathrm{C}^{5} \underline{\mathrm{H}}$, $\left.\mathrm{C}^{6} \underline{\mathrm{H}}, \mathrm{C}^{7} \underline{\mathrm{H}}\right), 7.66\left(\mathrm{~d}, 1 \mathrm{H},{ }^{3} J_{\mathrm{HH}}=7.1, \mathrm{C}^{8} \underline{\mathrm{H}}\right) . /{ }^{13} \mathrm{C}$ NMR $16.3\left(\mathrm{~d},{ }^{3} J_{\mathrm{CP}}=6.4, \underline{C}_{3} \mathrm{CH}_{2} \mathrm{O}\right), 21.7\left(\mathrm{~d},{ }^{4} J_{\mathrm{CP}}=2.2\right.$, $\left.\underline{\mathrm{C}}^{3} \mathrm{H}_{2}\right), 24.9\left(\mathrm{t},{ }^{3} J_{\mathrm{CF}}={ }^{3} J_{\mathrm{CP}}=9.2, \underline{\mathrm{C}}^{2} \mathrm{H}_{2}\right), 30.1\left(\mathrm{~s}, \underline{\mathrm{C}}^{4} \mathrm{H}_{2}\right), 63.5\left(\mathrm{~d},{ }^{2} J_{\mathrm{CP}}=5.9, \mathrm{CH}_{3} \underline{\mathrm{C}} \mathrm{H}_{2} \mathrm{O}\right), 125.8$ (s, $\underline{\mathrm{C}} \mathrm{H}$ of $\left.\mathrm{C}_{6} \mathrm{H}_{4}\right), 127.8\left(\mathrm{~s}, \underline{\mathrm{C} H}\right.$ of $\left.\mathrm{C}_{6} \mathrm{H}_{4}\right), 129.5\left(\mathrm{~s}, \underline{\mathrm{CH}}\right.$ of $\left.\mathrm{C}_{6} \mathrm{H}_{4}\right), 129.8\left(\mathrm{~s}, \underline{\mathrm{C}}^{8} \mathrm{H}^{\prime}\right.$ of $\left.\mathrm{C}_{6} \mathrm{H}_{4}\right), 130.5\left(\mathrm{~d},{ }^{4} J=6.7, \underline{\mathrm{C}}^{10}\right.$ of $\left.\mathrm{C}_{6} \mathrm{H}_{4}\right), 135.3\left(\mathrm{dd},{ }^{2} J=25.3,{ }^{2} J=13.7, \mathrm{P}(\mathrm{F}) \mathrm{C}=\underline{\mathrm{C}}\right), 140.5\left(\mathrm{~d},{ }^{3} J=4.4, \underline{\mathrm{C}}^{9}\right.$ of $\left.\mathrm{C}_{6} \mathrm{H}_{4}\right), 146.0\left(\mathrm{dd},{ }^{1} J=261.4,{ }^{1} J=240.0\right.$, $\mathrm{P}(\mathrm{F}) \underline{\mathrm{C}})$.

Diethyl 1-(2'-methyl)cyclohexylidene-1-fluoromethylphosphonate $6 \mathbf{s}(\boldsymbol{Z}): \mathrm{C}_{12} \mathrm{H}_{22} \mathrm{O}_{3} \mathrm{PF}(\mathrm{M}=264)$

$31 \mathbf{P} \mathbf{N M R}+6.6\left(\mathrm{~d},{ }^{2} J_{\mathrm{PF}}=111\right)$ / $1 \mathbf{1} \mathbf{H} \mathbf{N M R} 1.14\left(\mathrm{~d}, 3 \mathrm{H},{ }^{3} J_{\mathrm{HH}}=7.1, \mathrm{C}^{2} \mathrm{C}_{1}\right), 1.34\left(\mathrm{t}, 6 \mathrm{H},{ }^{3} J_{\mathrm{HH}}=7.0\right.$, $\left.\mathrm{C}_{3} \mathrm{CH}_{2} \mathrm{O}\right), 1.56\left(\mathrm{~m}, 4 \mathrm{H}, \mathrm{C}_{2}\right), 1.8-1.9\left(\mathrm{~m}, 3 \mathrm{H}, \mathrm{C}_{2}\right.$ et $\left.\mathrm{C}^{6} \underline{\mathrm{H}}_{\mathrm{A}} \mathrm{H}_{\mathrm{B}}\right), 2.80\left(\mathrm{dm}, 1 \mathrm{H},{ }^{2} J_{\mathrm{HH}}=15, \mathrm{C}^{6} \mathrm{H}_{\mathrm{A}} \underline{\mathrm{H}}_{\mathrm{B}}\right), 3.57$

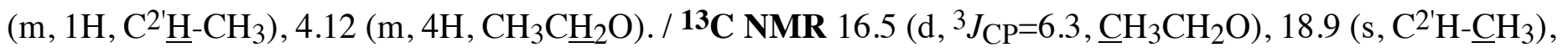


$20.5\left(\mathrm{~s}, \underline{\mathrm{CH}_{2}}\right), 21.8\left(\mathrm{t},{ }^{3} J_{\mathrm{CFcis}}=10.4,{ }^{3} J_{\mathrm{CPtrans}}=10.4, \underline{\mathrm{C}^{6}} \mathrm{H}_{2}\right), 27.3\left(\mathrm{~s}, \underline{\mathrm{CH}_{2}}\right), 28.9\left(\mathrm{~s}, \underline{\mathrm{C}^{\prime}} \mathrm{H}-\mathrm{CH}_{3}\right), 33.4\left(\mathrm{~s}, \underline{\mathrm{C}} \mathrm{H}_{2}\right)$, $62.8\left(\mathrm{~d},{ }^{2} J_{\mathrm{CP}}=4.5, \mathrm{CH}_{3} \underline{\mathrm{CH}_{2} \mathrm{O}}\right), 142.4\left(\mathrm{dd},{ }^{1} J=259.4,{ }^{1} J=236.5, \mathrm{P}(\mathrm{F}) \underline{\mathrm{C}}\right), 143.7\left(\mathrm{dd},{ }^{2} J=30.1,{ }^{2} J=4.7, \mathrm{P}(\mathrm{F}) \mathrm{C}=\underline{\mathrm{C}}\right)$.

Diethyl 1-(2'-methoxy)cyclohexylidene-1-fluoromethylphosphonate $\mathbf{6 t}: \mathrm{C}_{12} \mathrm{H}_{22} \mathrm{O}_{4} \mathrm{PF}(\mathrm{M}=280)$

E-isomer: ${ }^{31} \mathbf{P}$ NMR +4.6 (d, $\left.{ }^{2} J_{\mathrm{PF}}=107\right) . /{ }^{13} \mathbf{C}$ NMR $16.7\left(\mathrm{~d}, \underline{\mathrm{CH}_{3}} \mathrm{CH}_{2} \mathrm{O}\right), 20.4\left(\mathrm{~s}, \underline{\mathrm{CH}_{2}}\right), 22.1\left(\mathrm{~s}, \underline{\mathrm{C}}^{6} \mathrm{H}_{2}\right), 27.7$ (s, $\left.\underline{\mathrm{CH}}_{2}\right), 32.8$ (s, $\left.\underline{\mathrm{C}} \mathrm{H}_{2}\right), 56.0\left(\mathrm{~s}, \mathrm{O}^{\mathrm{C}} \mathrm{H}_{3}\right), 63.2\left(\mathrm{~d},{ }^{2} J_{\mathrm{CP}}=5.1, \mathrm{CH}_{3} \underline{\mathrm{CH}}_{2} \mathrm{O}\right), 71.1\left(\mathrm{t},{ }^{3} J_{\mathrm{CF}}={ }^{3} J_{\mathrm{CP}}=10.7, \underline{\mathrm{C}^{2}} \mathrm{H}-\right.$ $\left.\left.\mathrm{OCH}_{3}\right),=\mathrm{C}^{3} \mathrm{CH}_{3}\right), 137.7\left(\mathrm{dd},{ }^{2} J=28.5,3.9, \mathrm{P}(\mathrm{F}) \mathrm{C}=\underline{\mathrm{C}}^{1}\right), 145.0\left(\mathrm{dd},{ }^{1} J=264.7,{ }^{1} J=232.7, \mathrm{P}(\mathrm{F}) \underline{\mathrm{C}}\right)$.

Z-isomer: ${ }^{31} \mathbf{P} \mathbf{N M R}+4.6\left(\mathrm{~d},{ }^{2} J_{\mathrm{PF}}=107\right) . /{ }^{1} \mathbf{H} \mathbf{N M R} 1.34\left(\mathrm{t}, 6 \mathrm{H},{ }^{3} \mathrm{~J}_{\mathrm{HH}}=7.0, \mathrm{C}_{3} \mathrm{CH}_{2} \mathrm{O}\right), 1.2-2.1(\mathrm{~m}, 7 \mathrm{H}$, $\mathrm{C}^{6} \underline{\mathrm{H}}_{\mathrm{A}} \mathrm{H}_{\mathrm{B}}$ et $\left.\underline{\mathrm{C}}_{2}\right), 2.73\left(\mathrm{dm}, 1 \mathrm{H},{ }^{2} J_{\mathrm{HH}}=12.0, \mathrm{C}^{6} \mathrm{H}_{\mathrm{A}} \underline{\mathrm{H}}_{\mathrm{B}}\right), 3.26\left(\mathrm{~s}, 3 \mathrm{H}, \mathrm{OC} \underline{\mathrm{H}}_{3}\right), 4.13\left(\mathrm{~m}, 4 \mathrm{H}, \mathrm{CH}_{3} \mathrm{C}_{2} \mathrm{O}\right), 4.93$ $\left(\mathrm{m}, 1 \mathrm{H}, J=3.1, \mathrm{C}^{2} \underline{\mathrm{H}}-\mathrm{OCH}_{3}\right) . /{ }^{13} \mathbf{C}$ NMR $16.5\left(\mathrm{~d},{ }^{3} J_{\mathrm{CP}}=6.3, \underline{\mathrm{CH}}_{3} \mathrm{CH}_{2} \mathrm{O}\right), 20.2\left(\mathrm{~s}, \underline{\mathrm{CH}}_{2}\right), 22.2$ (t, $\left.{ }^{3} J_{\mathrm{CF}}={ }^{3} J_{\mathrm{CP}}=9.4, \underline{\mathrm{C}}^{6} \mathrm{H}_{2}\right), 27.1\left(\mathrm{~s}, \underline{\mathrm{C}} \mathrm{H}_{2}\right), 33.4\left(\mathrm{~s}, \underline{\mathrm{C}} \mathrm{H}_{2}\right), 55.7\left(\mathrm{~s}, \mathrm{OCH}_{3}\right), 63.1\left(\mathrm{~d},{ }^{2} J_{\mathrm{CP}}=4.9, \mathrm{CH}_{3} \underline{\mathrm{CH}}_{2} \mathrm{O}\right), 72.1(\mathrm{~d}$ $\left.\left.{ }^{3} J_{\mathrm{CF}}={ }^{3} J_{\mathrm{CP}}=6.3, \underline{\mathrm{C}}^{2} \mathrm{H}-\mathrm{OCH}_{3}\right),=\mathrm{C}^{3} \mathrm{CH}_{3}\right), 138.8\left(\mathrm{dd},{ }^{2} J=29.3,4.6, \mathrm{P}(\mathrm{F}) \mathrm{C}=\underline{\mathrm{C}}^{1}\right), 145.1\left(\mathrm{dd},{ }^{1} J=266.4,{ }^{1} J=233.4\right.$, $\mathrm{P}(\mathrm{F}) \underline{\mathrm{C}}$. / / m/z (EI) 280 (M+ , 6), 265 (100), 237 (35), 209 (50).

1-Fluoro 2-(4'-methoxy)phenyl-1-(triisopropyl)silyl-ethene 7 (E) $\mathrm{C}_{18} \mathrm{H}_{29} \mathrm{OSiF}(\mathrm{M}=308)$ : The same procedure was carried out using chlorotriisopropylsilane $(0.85 \mathrm{~g}, 4.4 \mathrm{mmol})$ instead of chlorotrimethylsilane and anisaldehyde $(0.55 \mathrm{~g}, 4 \mathrm{mmol})$ as carbonyl compound. The crude product was purified by a column chromatography with the same silica gel (eluant : hexane).

1H NMR 1.0-1.3 (m, 21H, $\left.\left(\mathrm{C}_{3}\right)_{2} \mathrm{C} \underline{\mathrm{H}}\right), 3.82\left(\mathrm{~s}, 3 \mathrm{H}, \mathrm{OC}_{3}\right), 5.80\left(\mathrm{~d}, 1 \mathrm{H},{ }^{3} J_{\mathrm{HF}}=53.8, \mathrm{FC}=\mathrm{C} \underline{\mathrm{H}}\right), 6.87(\mathrm{~d}, 2 \mathrm{H}$, ${ }^{3} J_{\mathrm{HH}}=8.8, \underline{\mathrm{H}}_{\text {meta }}$ of $\left.\mathrm{C}_{6} \mathrm{H}_{4}\right), 7.54\left(\mathrm{~d}, 2 \mathrm{H},{ }^{3} \mathrm{~J}_{\mathrm{HH}}=8.8, \underline{\mathrm{H}}_{\text {ortho }}\right.$ of $\left.\mathrm{C}_{6} \mathrm{H}_{4}\right) . /{ }^{13} \mathrm{C}$ NMR $11.3\left(\mathrm{~s},\left(\mathrm{CH}_{3}\right)_{2} \underline{\mathrm{CH}}\right), 19.1(\mathrm{~s}$, $\left.\left(\underline{\mathrm{CH}}_{3}\right)_{2} \mathrm{CH}\right), 55.7\left(\mathrm{~s}, \mathrm{O}_{\underline{C}}{ }_{3}\right), 114.4\left(\mathrm{~s}, \underline{\mathrm{C}}_{\text {meta }}\right.$ of $\left.\mathrm{C}_{6} \mathrm{H}_{4}\right), 122.6(\mathrm{~s}, \mathrm{Si}(\mathrm{F}) \mathrm{C}=\mathrm{C} \underline{\mathrm{H}}), 127.3\left(\mathrm{~d},{ }^{2} J_{\mathrm{CF}}=2.8, \underline{\mathrm{C}}_{\mathrm{ipso}}\right.$ of $\left.\mathrm{C}_{6} \mathrm{H}_{4}\right), 131.2\left(\mathrm{~d},{ }^{4} J_{\mathrm{CF}}=8.0, \underline{\mathrm{C}}_{\text {ortho }}\right.$ of $\left.\mathrm{C}_{6} \mathrm{H}_{4}\right), 159.5\left(\mathrm{~s}, \underline{\mathrm{C}}_{\text {para }}\right.$ of $\left.\mathrm{C}_{6} \mathrm{H}_{4}\right), 166.5\left(\mathrm{~d},{ }^{1} J_{\mathrm{CF}}=290.0, \mathrm{Si}(\mathrm{F}) \underline{\mathrm{C}}\right) . / \mathrm{m} / \mathbf{z}$ (EI) $308\left(\mathrm{M}^{+}, 28\right), 223(24), 208(10), 195$ (12).

Diethyl 1-chloro-2-(2'-furyl)ethenephosphonate $8 \mathrm{C}_{10} \mathrm{H}_{14} \mathrm{O}_{4} \mathrm{PCl}(\mathrm{M}=264)$ : The same procedure was carried out using diethyl 1,1,1-trichloromethylphosphonate (1.13 g, $4.4 \mathrm{mmol})$ instead of diethyl 1-bromo-1,1difluoromethylphosphonate and furaldehyde $(0.39 \mathrm{~g}, 4 \mathrm{mmol})$ as carbonyl compound. The crude product was purified by a column chromatography with the same silica gel (eluant : hexane / AcOEt : 85 / 15).

Z-isomer: ${ }^{31} \mathbf{P}$ NMR +10.8 (s). / $1 \mathbf{H}$ NMR $1.36\left(\mathrm{t}, 6 \mathrm{H},{ }^{3} \mathrm{~J}_{\mathrm{HH}}=7.1, \mathrm{C}_{3} \mathrm{CH}_{2} \mathrm{O}\right), 4.15\left(\mathrm{~m}, 4 \mathrm{H}, \mathrm{CH}_{3} \mathrm{CH}_{2} \mathrm{O}\right), 6.53$ $\left(\mathrm{ddd}, 1 \mathrm{H},{ }^{3} J_{\mathrm{HH}}=3.5,{ }^{3} J_{\mathrm{HH}}=1.8, J=0.5, \underline{\mathrm{H}}^{4}\right.$ of $\left.\mathrm{C}_{4} \mathrm{H}_{3} \mathrm{O}\right), 7.18\left(\mathrm{~d}, 1 \mathrm{H},{ }^{3} J_{\mathrm{HH}}=3.5, \underline{\mathrm{H}}^{3}\right.$ of $\left.\mathrm{C}_{4} \mathrm{H}_{3} \mathrm{O}\right), 7.54(\mathrm{~m}, 1 \mathrm{H}$, $\underline{\mathrm{H}}^{5^{\prime}}$ of $\left.\mathrm{C}_{4} \mathrm{H}_{3} \mathrm{O}\right), 7.62\left(\mathrm{~d}, 1 \mathrm{H},{ }^{3} J_{\mathrm{HP}}=14.4, \mathrm{P}(\mathrm{Cl}) \mathrm{C}=\mathrm{CH}\right) . /{ }^{13} \mathbf{C} \mathbf{N M R} 16.3\left(\mathrm{~d},{ }^{3} J_{\mathrm{CP}}=7.0, \underline{\mathrm{CH}}_{3} \mathrm{CH}_{2} \mathrm{O}\right), 63.3(\mathrm{~d}$, $\left.{ }^{2} J_{\mathrm{CP}}=4.9, \mathrm{CH}_{3} \underline{\mathrm{CH}} \mathrm{H}_{2} \mathrm{O}\right), 112.5\left(\mathrm{~s}, \underline{\mathrm{C}}^{3}\right.$ of $\left.\mathrm{C}_{4} \mathrm{H}_{3} \mathrm{O}\right), 116.2$ (s, $\underline{\mathrm{C}}^{4}$ of $\left.\mathrm{C}_{4} \mathrm{H}_{3} \mathrm{O}\right), 117.0\left(\mathrm{~d},{ }^{1} J_{\mathrm{CP}}=217.2, \mathrm{P}(\mathrm{Cl}) \underline{\mathrm{C}}\right)$, $129.9\left(\mathrm{~d},{ }^{2} J_{\mathrm{CP}}=21.3, \mathrm{P}(\mathrm{Cl}) \mathrm{C}=\underline{\mathrm{CH}}\right), 144.5\left(\mathrm{~s}, \underline{\mathrm{C}}^{\prime}\right.$ of $\left.\mathrm{C}_{4} \mathrm{H}_{3} \mathrm{O}\right), 149.1\left(\mathrm{~d},{ }^{3} J_{\mathrm{CP}}=21.5, \underline{\mathrm{C}}^{2}\right.$ of $\left.\mathrm{C}_{4} \mathrm{H}_{3} \mathrm{O}\right) . / \mathbf{~ m} / \mathbf{z}(\mathbf{E I})$ $266\left(\mathrm{M}^{+37} \mathrm{Cl}, 8\right), 264\left(\mathrm{M}^{+35} \mathrm{Cl}, 24\right), 229$ (93), $201(35), 173$ (100).

E-isomer: ${ }^{31} \mathbf{P}$ NMR +7.8 (s). / ${ }^{\mathbf{1}} \mathbf{H}$ NMR $1.32\left(\mathrm{t}, 6 \mathrm{H},{ }^{3} J_{\mathrm{HH}}=7.1, \mathrm{C}_{3} \mathrm{CH}_{2} \mathrm{O}\right), 4.15\left(\mathrm{dqd}, 4 \mathrm{H},{ }^{3} J_{\mathrm{HP}}=7.9\right.$, $\left.{ }^{3} J_{\mathrm{HH}}=7.1, J=3.2, \mathrm{CH}_{3} \underline{\mathrm{C}}_{2} \mathrm{O}\right), 6.44\left(\mathrm{ddd}, 1 \mathrm{H},{ }^{3} J_{\mathrm{HH}}=3.6,{ }^{3} J_{\mathrm{HH}}=1.8,{ }^{5} J_{\mathrm{HH}}=0.5, \underline{\mathrm{H}}^{4}\right.$ of $\left.\mathrm{C}_{4} \mathrm{H}_{3} \mathrm{O}\right), 7.33(\mathrm{dd}, 1 \mathrm{H}$, $\left.{ }^{3} J_{\mathrm{HP}}=35.2,{ }^{5} J_{\mathrm{HH}}=0.5, \mathrm{P}(\mathrm{Cl}) \mathrm{C}=\mathrm{C} \underline{\mathrm{H}}\right), 7.35\left(\mathrm{dd} 1 \mathrm{H},{ }^{3} J_{\mathrm{HH}}=3.6,{ }^{4} J_{\mathrm{HH}}=0.6, \underline{\mathrm{H}}^{3}\right.$ of $\left.\mathrm{C}_{4} \mathrm{H}_{3} \mathrm{O}\right), 7.45(\mathrm{dd}, 1 \mathrm{H}$,

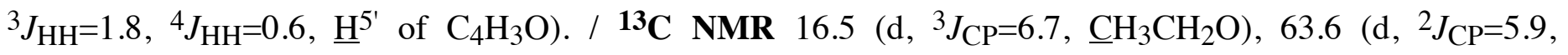
$\left.\mathrm{CH}_{3} \underline{\mathrm{CH}}_{2} \mathrm{O}\right), 112.9\left(\mathrm{~s}, \underline{\mathrm{C}}^{3}\right.$ of $\left.\mathrm{C}_{4} \mathrm{H}_{3} \mathrm{O}\right), 116.0\left(\mathrm{~s}, \underline{\mathrm{C}}^{4}\right.$ of $\left.\mathrm{C}_{4} \mathrm{H}_{3} \mathrm{O}\right), 118.4\left(\mathrm{~d},{ }^{1} J_{\mathrm{CP}}=208.6, \mathrm{P}(\mathrm{Cl}) \underline{\mathrm{C}}\right), 133.3(\mathrm{~d}$, $\left.{ }^{2} J_{\mathrm{CP}}=15.3, \mathrm{P}(\mathrm{Cl}) \mathrm{C}=\underline{\mathrm{CH}}\right), 144.5\left(\mathrm{~s}, \underline{\mathrm{C}}^{5^{\prime}}\right.$ of $\left.\mathrm{C}_{4} \mathrm{H}_{3} \mathrm{O}\right), 148.8\left(\mathrm{~d},{ }^{3} J_{\mathrm{CP}}=4.6, \underline{\mathrm{C}}^{2}\right.$ of $\left.\mathrm{C}_{4} \mathrm{H}_{3} \mathrm{O}\right) . / \mathbf{m} / \mathbf{z}(\mathbf{E I}) 266\left(\mathrm{M}^{+}\right.$ $\left.{ }^{37} \mathrm{Cl}, 6\right), 264\left(\mathrm{M}^{+35} \mathrm{Cl}, 20\right), 229$ (71), 201 (32), 173 (100). 
Diethyl 2-(2'-furyl)ethenephosphonate $9 \mathrm{C}_{10} \mathrm{H}_{15} \mathrm{O}_{4} \mathrm{P}(\mathrm{M}=230)$ : The previously described procedure ${ }^{13}$ was used with diethyl methylphosphonate as starting material.

E-isomer: ${ }^{31} \mathbf{P}$ NMR +16.3 (s). / ${ }^{1} \mathbf{H}$ NMR $1.36\left(\mathrm{t}, 6 \mathrm{H},{ }^{3} J_{\mathrm{HH}}=7.1, \mathrm{CH}_{3} \mathrm{CH}_{2} \mathrm{O}\right), 4.13\left(\mathrm{dq}, 4 \mathrm{H},{ }^{3} J_{\mathrm{HP}}=7.9\right.$, $\left.{ }^{3} J_{\mathrm{HH}}=7.1, \mathrm{CH}_{3} \underline{\mathrm{H}}_{2} \mathrm{O}\right), 6.12\left(\mathrm{dd}, 3 \mathrm{H},{ }^{2} J_{\mathrm{HP}}=17.8,{ }^{3} J_{\mathrm{HH}}=17.2, \mathrm{P}(\underline{\mathrm{H}}) \mathrm{C}\right), 6.47\left(\mathrm{dd}, 1 \mathrm{H},{ }^{3} J_{\mathrm{HH}}=3.6,{ }^{3} J_{\mathrm{HH}}=1.8, \underline{\mathrm{H}}^{4}\right.$ of $\left.\mathrm{C}_{4} \mathrm{H}_{3} \mathrm{O}\right), 6.56\left(\mathrm{~d}_{\text {broad, }} 1 \mathrm{H},{ }^{3} J_{\mathrm{HP}}=3.6, \underline{\mathrm{H}}^{3}\right.$ of $\left.\mathrm{C}_{4} \mathrm{H}_{3} \mathrm{O}\right), 7.27\left(\mathrm{dd}, 1 \mathrm{H},{ }^{3} J_{\mathrm{HP}}=22.5,{ }^{3} J_{\mathrm{HH}}=17.2, \mathrm{P}(\mathrm{H}) \mathrm{C}=\mathrm{C} \underline{\mathrm{H}}\right)$, $7.48\left(\mathrm{~d}_{\text {broad }}, 1 \mathrm{H},{ }^{3} J_{\mathrm{HH}}=0.5, \underline{\mathrm{H}}^{5}\right.$ of $\left.\mathrm{C}_{4} \mathrm{H}_{3} \mathrm{O}\right) . /{ }^{13} \mathrm{C}$ NMR $16.9\left(\mathrm{~d},{ }^{3} J_{\mathrm{CP}}=6.3, \underline{\mathrm{CH}}_{3} \mathrm{CH}_{2} \mathrm{O}\right), 62.3\left(\mathrm{~d},{ }^{2} J_{\mathrm{CP}}=4.6\right.$, $\left.\mathrm{CH}_{3} \underline{\mathrm{CH}}_{2} \mathrm{O}\right), 111.6\left(\mathrm{~d},{ }^{1} J_{\mathrm{CP}}=193.8, \mathrm{P}(\mathrm{H}) \underline{\mathrm{C}}\right), 112.4\left(\mathrm{~s}, \underline{\mathrm{C}}^{4}\right.$ of $\left.\mathrm{C}_{4} \mathrm{H}_{3} \mathrm{O}\right), 114.5$ (s, $\underline{\mathrm{C}}^{3}$ of $\left.\mathrm{C}_{4} \mathrm{H}_{3} \mathrm{O}\right), 135.8(\mathrm{~d}$, $\left.{ }^{2} J_{\mathrm{CP}}=7.6, \mathrm{PC}=\underline{\mathrm{CH}}\right), 145.1\left(\mathrm{~s}, \underline{\mathrm{C}}^{5}\right.$ of $\left.\mathrm{C}_{4} \mathrm{H}_{3} \mathrm{O}\right), 151.5\left(\mathrm{~d},{ }^{3} J_{\mathrm{CP}}=26.0, \underline{\mathrm{C}}^{2}\right.$ of $\left.\mathrm{C}_{4} \mathrm{H}_{3} \mathrm{O}\right) . / \mathbf{m} / \mathbf{z}(\mathbf{E I}) 230\left(\mathrm{M}^{+}, 100\right)$, 202 (22), 185 (17), 174 (43).

Z-isomer: ${ }^{31} \mathbf{P} \mathbf{N M R}+20.1$ (s). / $\mathbf{1} \mathbf{H}$ NMR $1.32\left(\mathrm{t}, 6 \mathrm{H},{ }^{3} J_{\mathrm{HH}}=7.1, \mathrm{CH}_{3} \mathrm{CH}_{2} \mathrm{O}\right), 4.13\left(\mathrm{dq}, 4 \mathrm{H},{ }^{3} J_{\mathrm{HP}}=7.9\right.$, $\left.{ }^{3} J_{\mathrm{HH}}=7.1, \mathrm{CH}_{3} \underline{\mathrm{C}}_{2} \mathrm{O}\right), 5.58\left(\mathrm{t}, 1 \mathrm{H},{ }^{2} J_{\mathrm{HP}}={ }^{3} J_{\mathrm{HH}}=14.6, \mathrm{P}(\underline{\mathrm{H}}) \mathrm{C}\right), 6.49\left(\mathrm{dd}, 1 \mathrm{H},{ }^{3} J_{\mathrm{HH}}=3.6,{ }^{3} J_{\mathrm{HH}}=1.8, \underline{\mathrm{H}}^{4}\right.$ of $\left.\mathrm{C}_{4} \mathrm{H}_{3} \mathrm{O}\right), 7.03\left(\mathrm{dd}, 1 \mathrm{H},{ }^{3} J_{\mathrm{HP}}=49.2,{ }^{3} J_{\mathrm{HH}}=14.6, \mathrm{PC}=\mathrm{C} \underline{\mathrm{H}}\right), 7.22\left(\mathrm{~d}, 1 \mathrm{H},{ }^{3} J_{\mathrm{HH}}=3.6, \underline{\mathrm{H}}^{3}\right.$ of $\left.\mathrm{C}_{4} \mathrm{H}_{3} \mathrm{O}\right), 7.51\left(\mathrm{~d}_{\text {broad }}\right.$,

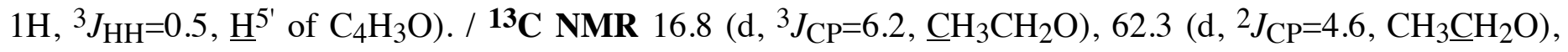
$111.8\left(\mathrm{~d},{ }^{1} J_{\mathrm{CP}}=188.0, \mathrm{P}(\mathrm{H}) \underline{\mathrm{C}}\right), 112.9\left(\mathrm{~s}, \underline{\mathrm{C}}^{4}\right.$ of $\left.\mathrm{C}_{4} \mathrm{H}_{3} \mathrm{O}\right), 115.9\left(\mathrm{~s}, \underline{\mathrm{C}}^{3}\right.$ of $\left.\mathrm{C}_{4} \mathrm{H}_{3} \mathrm{O}\right), 134.7(\mathrm{~s}, \mathrm{PC}=\underline{\mathrm{C}} \mathrm{H}), 144.7(\mathrm{~s}$, $\underline{\mathrm{C}}^{5}$ of $\left.\mathrm{C}_{4} \mathrm{H}_{3} \mathrm{O}\right), 151.1\left(\mathrm{~d},{ }^{3} J_{\mathrm{CP}}=10.6, \underline{\mathrm{C}}^{2}\right.$ of $\left.\mathrm{C}_{4} \mathrm{H}_{3} \mathrm{O}\right) . / \mathbf{m} / \mathbf{z}(\mathbf{E I}) 230\left(\mathrm{M}^{+}, 65\right), 202$ (13), 185 (5), 174 (26).

Diethyl-1-methyl 2-(2'-furyl)ethenephosphonate $10 \mathrm{C}_{11} \mathrm{H}_{17} \mathrm{O}_{4} \mathrm{P}(\mathrm{M}=244)$ : The general procedure for synthesis of 6 was carried out using diethyl ethylphosphonate $(0.73 \mathrm{~g}, 4.4 \mathrm{mmol})$ instead of diethyl 1-bromo1,1-difluoromethylphosphonate and furaldehyde $(0.39 \mathrm{~g}, 4 \mathrm{mmol})$ as carbonyl compound. The crude product was purified by a column chromatography with the same silica gel (eluant : hexane / AcOEt : 70 / 30).

E-isomer: ${ }^{31} \mathbf{P}$ NMR +22.8 (s). / $1 \mathbf{H}$ NMR $1.31\left(\mathrm{t}, 6 \mathrm{H},{ }^{3} J_{\mathrm{HH}}=7.1, \mathrm{CH}_{3} \mathrm{CH}_{2} \mathrm{O}\right), 2.14\left(\mathrm{dd}, 3 \mathrm{H},{ }^{3} J_{\mathrm{HP}}=13.4\right.$, $\left.{ }^{4} J_{\mathrm{HH}}=1.1, \mathrm{P}(\underline{\mathrm{H}} 3 \mathrm{C}) \mathrm{C}\right), 4.06\left(\mathrm{dqd}, 4 \mathrm{H},{ }^{3} J_{\mathrm{HP}}=7.5,{ }^{3} J_{\mathrm{HH}}=7.1,5 J_{\mathrm{HF}}=3.5, \mathrm{CH}_{3} \underline{\mathrm{H}}_{2} \mathrm{O}\right), 6.45\left(\mathrm{dd}, 1 \mathrm{H},{ }^{3} J_{\mathrm{HH}}=3.3\right.$, ${ }^{3} J_{\mathrm{HH}}=1.8, \underline{\mathrm{H}}^{4}$ of $\left.\mathrm{C}_{4} \mathrm{H}_{3} \mathrm{O}\right), 6.53\left(\mathrm{~d}, 1 \mathrm{H},{ }^{3} J_{\mathrm{HP}}=3.3, \mathrm{P}\left(\mathrm{H}_{3} \mathrm{C}\right) \mathrm{C}=\mathrm{C} \underline{\mathrm{H}}\right), 7.12\left(\mathrm{~s}, 1 \mathrm{H}, \underline{\mathrm{H}}^{3}\right.$ of $\left.\mathrm{C}_{4} \mathrm{H}_{3} \mathrm{O}\right), 7.48(\mathrm{~d}, 1 \mathrm{H}$, ${ }^{3} J_{\mathrm{HH}}=0.6, \underline{\mathrm{H}}^{5}$ of $\left.\mathrm{C}_{4} \mathrm{H}_{3} \mathrm{O}\right) . /{ }^{13} \mathrm{C}$ NMR $14.9\left(\mathrm{~d},{ }^{2} J_{\mathrm{CP}}=7.7, \mathrm{P}\left(\mathrm{H}_{3} \underline{\mathrm{C}}\right) \mathrm{C}\right), 16.8\left(\mathrm{~d},{ }^{3} J_{\mathrm{CP}}=6.1, \underline{\mathrm{CH}}_{3} \mathrm{CH}_{2} \mathrm{O}\right), 62.1(\mathrm{~d}$, $\left.{ }^{2} J_{\mathrm{CP}}=4.8, \mathrm{CH}_{3} \underline{\mathrm{CH}} \mathrm{H}_{2} \mathrm{O}\right), 112.2\left(\mathrm{~s}, \underline{\mathrm{C}}^{4}\right.$ of $\left.\mathrm{C}_{4} \mathrm{H}_{3} \mathrm{O}\right), 114.7\left(\mathrm{~s}, \underline{\mathrm{C}}^{3}\right.$ of $\left.\mathrm{C}_{4} \mathrm{H}_{3} \mathrm{O}\right), 122.4\left(\mathrm{~d},{ }^{1} J_{\mathrm{CP}}=181.6, \mathrm{P}\left(\mathrm{H}_{3} \mathrm{C}\right) \underline{\mathrm{C}}\right)$, $130.1\left(\mathrm{~d},{ }^{2} J_{\mathrm{CP}}=13.7, \mathrm{PC}=\underline{\mathrm{CH}}\right), 144.2\left(\mathrm{~s}, \underline{\mathrm{C}}^{5}\right.$ of $\left.\mathrm{C}_{4} \mathrm{H}_{3} \mathrm{O}\right), 152.3\left(\mathrm{~d},{ }^{3} J_{\mathrm{CP}}=29.0, \underline{\mathrm{C}}^{2}\right.$ of $\left.\mathrm{C}_{4} \mathrm{H}_{3} \mathrm{O}\right) . / \mathrm{m} / \mathbf{z}(\mathbf{E I}) 244$ $\left(\mathrm{M}^{+}, 64\right), 216(29), 188(46), 171(23)$.

Z-isomer: 31P NMR+17.8 (s). / ${ }^{1} \mathbf{H}$ NMR $1.27\left(\mathrm{t}, 6 \mathrm{H},{ }^{3} J_{\mathrm{HH}}=7.0, \mathrm{CH}_{3} \mathrm{CH}_{2} \mathrm{O}\right), 2.10\left(\mathrm{dd}, 3 \mathrm{H},{ }^{3} J_{\mathrm{HP}}=13.1\right.$, $\left.{ }^{4} J_{\mathrm{HH}}=1.7, \mathrm{P}\left(\underline{\mathrm{H}}_{3} \mathrm{C}\right) \mathrm{C}\right), 4.07\left(\mathrm{dqd}, 4 \mathrm{H},{ }^{3} J_{\mathrm{HP}}=7.9,{ }^{3} J_{\mathrm{HH}}=7.0,{ }^{5} J_{\mathrm{HF}}=1.3, \mathrm{CH}_{3} \mathrm{C}_{2} \mathrm{O}\right), 6.41\left(\mathrm{ddd}, 1 \mathrm{H},{ }^{3} J_{\mathrm{HH}}=3.6\right.$, ${ }^{3} J_{\mathrm{HH}}=1.7,{ }^{5} J_{\mathrm{HH}}=0.5, \underline{\mathrm{H}}^{4}$ of $\left.\mathrm{C}_{4} \mathrm{H}_{3} \mathrm{O}\right), 6.88\left(\mathrm{dm}, 1 \mathrm{H},{ }^{3} J_{\mathrm{HP}}=46.4,{ }^{4} J_{\mathrm{HH}}=1.7, \mathrm{PC}=\mathrm{C} \underline{\mathrm{H}}\right), 7.14\left(\mathrm{~d}, 1 \mathrm{H},{ }^{3} J_{\mathrm{HH}}=3.6\right.$, $\underline{\mathrm{H}}^{3}$ of $\left.\mathrm{C}_{4} \mathrm{H}_{3} \mathrm{O}\right), 7.40\left(\mathrm{~d}_{\text {broad }}, 1 \mathrm{H},{ }^{3} J_{\mathrm{HH}}=1.7, \underline{\mathrm{H}}^{5}\right.$ of $\left.\mathrm{C}_{4} \mathrm{H}_{3} \mathrm{O}\right) . /{ }^{13} \mathrm{C}$ NMR $16.7\left(\mathrm{~d},{ }^{3} J_{\mathrm{CP}}=7.1, \underline{\mathrm{CH}}_{3} \mathrm{CH}_{2} \mathrm{O}\right), 24.3$ $\left(\mathrm{d},{ }^{2} J_{\mathrm{CP}}=10.7, \mathrm{P}\left(\mathrm{H}_{3} \underline{\mathrm{C}}\right) \mathrm{C}\right), 62.2\left(\mathrm{~d},{ }^{2} J_{\mathrm{CP}}=5.3, \mathrm{CH}_{3} \underline{\mathrm{CH}_{2} \mathrm{O}}\right), 112.5\left(\mathrm{~s}, \underline{\mathrm{C}}^{4}\right.$ of $\left.\mathrm{C}_{4} \mathrm{H}_{3} \mathrm{O}\right), 113.8\left(\mathrm{~s}, \underline{\mathrm{C}}^{3}\right.$ of $\left.\mathrm{C}_{4} \mathrm{H}_{3} \mathrm{O}\right)$, $122.2\left(\mathrm{~d},{ }^{1} J_{\mathrm{CP}}=176.8, \mathrm{P}\left(\mathrm{H}_{3} \mathrm{C}\right) \underline{\mathrm{C}}\right), 131.7\left(\mathrm{~d},{ }^{2} J_{\mathrm{CP}}=7.9, \mathrm{PC}=\underline{\mathrm{CH}}\right), 143.3\left(\mathrm{~s}, \underline{\mathrm{C}}^{5}\right.$ of $\left.\mathrm{C}_{4} \mathrm{H}_{3} \mathrm{O}\right), 150.8\left(\mathrm{~d},{ }^{3} J_{\mathrm{CP}}=8.0\right.$, $\underline{\mathrm{C}}^{2}$ of $\left.\mathrm{C}_{4} \mathrm{H}_{3} \mathrm{O}\right) . /$ m/z (EI) $244\left(\mathrm{M}^{+}, 39\right), 216$ (19), 188 (36), 171 (17).

\section{ACKNOWLEDGEMENTS}

Support of this work by the CNRS for J. C. and R. W. is gratefully acknowledged. We are also grateful to Mr. M. Levard (URA 1307) of the Ecole Polytechnique for the mass spectra. 


\section{REFERENCES \& NOTES}

1. Engel, R. Chem. Rev. 1977, 77, 349.

2. a) Blackburn, G. M.; Eckstein, F.; Kent, D. E.; Perrée, T. D. Nucleosides and Nucleotides 1985, 4, 165.

b) Blackburn, G. M.; Kent, D. E. J. Chem. Soc., Perkin Trans. 1 1986, 913. c) Blackburn, G. M.; Parrat, M. J. ibid. 1986, 1425. d) Blackburn, G. M.; Rashid, A. J. Chem. Soc., Chem. Comm. 1988, 317. e) Chambers, R. D.; Jaouhari, R.; O'Hagan, D. Tetrahedron 1989, 45, 5101 . f) Thatcher, G. R. J.; Campbell, A. S. J. Org. Chem. 1993, 58, 2272.

3. Gross, R. S.; Mehdi, S., McCarthy, J. R. Tetrahedron Lett. 1993, 34, 7197.

4. Patois, C.; Savignac, P. J. Chem. Soc., Chem. Comm. 1993, 1711.

5. Johnson, A. W.; Kaska, W. C.; Ostoja Starzewski, K. A.; Dixon, D. A. in Ylides and Imines of Phosphorus, Johnson, A. W., Ed., John Wiley \& Sons, Inc., 1993.

6. a) Peterson, D. J. J. Org. Chem. 1968, 33, 780. b) Carey, F. A.; Court, A. S. J. Org. Chem. 1972, $37,939$.

c) Trindle, C.; Hwang, J. T.; Carey, F. A. J. Org. Chem. 1973, 38, 2664. d) Ager, D. J. Synthesis 1984, 384.

7. $\quad$ Bakkas, S.; Juliard, M.; Chanon, M. Tetrahedron 1987, 43, 501.

8. Burton, D. J.; Flynn, R. M. J. Fluorine Chem. 1977, 10, 329.

9. a) Hudrlik, P. F.; Peterson, D.; Rona, R. J. J. Org. Chem. 1975, 40, 2163. b) Hudrlik, P. F.; Peterson, D. J. Am. Chem. Soc. 1975, 97, 1464. c) Hudrlik, P. F.; Agwaranmgbo, E. L. O.; Hudrlik, A. M. J. Org. Chem. 1989, 54, 5613.

10. a) Sakurai, H.; Nishiwaki, K. I.; Kira, M. Tetrahedron Lett. 1973, 4193. b) Seyferth, D.; Lefferts, J. L.; Lambert, R. L. Jr. J. Organomet. Chem. 1977, 142, 39.

11. a) Hudrlik, P. F.; Peterson, D. Tetrahedron Lett. 1972, 1785. b) Utimoto, K.; Obayashi, M.; Nozaki, H. J. Org. Chem. 1976, 41, 2940.

12. Armstrong, D. R.; Barr, D.; Davidson, M. G.; Hutton, G.; O'Brien, P.; Snaith, R.; Warren, S. J. Organomet. Chem. 1996, to be published.

13. Aboujaoude, E. E.; Liétjé, S.; Collignon, N.; Teulade, M. P.; Savignac, P. Synthesis 1986, 934.

This work was first presented at the "Journées de Chimie Organique" (JCO 95), 12-15 september 1995, Ecole Polytechnique, Palaiseau, France.

We gratefuly acknowledge Dr O'Hagan of Durham University for his communication to us on the subject of his publication recently accepted by The Journal of Fluorine Chemistry. 